\title{
Pyruvate controls the checkpoint inhibitor PD-L1 and suppresses T cell immunity
}

\author{
Ryu Watanabe, ${ }^{1}$ Tsuyoshi Shirai, ${ }^{1,2}$ Hong Namkoong, ${ }^{1}$ Hui Zhang, ${ }^{1}$ Gerald J. Berry, ${ }^{3}$ Barbara B. Wallis, ${ }^{1}$ Benedikt Schaefgen, ${ }^{1}$ \\ David G. Harrison, ${ }^{4}$ Jennifer A. Tremmel, ${ }^{5}$ John C. Giacomini, ${ }^{5}$ Jörg J. Goronzy, ${ }^{1}$ and Cornelia M. Weyand ${ }^{1}$
}

'Division of Immunology and Rheumatology, Department of Medicine, Stanford University School of Medicine, Stanford, California, USA. ²Department of Hematology and Rheumatology, Tohoku University Graduate School of Medicine, Sendai, Japan. ${ }^{3}$ Department of Pathology, Stanford University School of Medicine, Stanford, California, USA. ${ }^{4}$ Division of Clinical Pharmacology, Department of Medicine, Vanderbilt University School of Medicine, Nashville, Tennessee, USA. 'Division of Cardiovascular Medicine, Department of Medicine, Stanford University School of Medicine, Stanford, California, USA.

\begin{abstract}
Patients with coronary artery disease (CAD) are at high risk for reactivation of the varicella zoster virus (VZV) and development of herpes zoster $(\mathrm{HZ})$. Here, we found that macrophages from patients with CAD actively suppress $\mathrm{T}$ cell activation and expansion, leading to defective VZV-specific T cell immunity. Monocyte-derived and plaque-infiltrating macrophages from patients with CAD spontaneously expressed high surface density of the immunoinhibitory ligand programmed death ligand-1 (PD-L1), thereby providing negative signals to programmed death-1+ (PD-1+) T cells. We determined that aberrant PD-L1 expression in patient-derived macrophages was metabolically controlled. Oversupply of the glycolytic intermediate pyruvate in mitochondria from CAD macrophages promoted expression of PD-L1 via induction of the bone morphogenetic protein 4/phosphorylated SMAD1/5/IFN regulatory factor 1 (BMP4/p-SMAD1/5/IRF1) signaling pathway. Thus, CAD macrophages respond to nutrient excess by activating the immunoinhibitory PD-1/PD-L1 checkpoint, leading to impaired T cell immunity. This finding indicates that metabolite-based immunotherapy may be a potential strategy for restoring adaptive immunity in CAD.
\end{abstract}

\section{Introduction}

Varicella zoster virus (VZV) causes chickenpox in young and herpes zoster (HZ; shingles) in older individuals $(1,2)$. Like all herpes viruses, VZV enters latency, with sensory ganglia serving as reservoirs. The virus is kept dormant by intact antiviral immunity, specifically virus-specific CD4 T cells (3). In the general population, the lifetime risk for developing viral reactivation presenting as the typically painful, blistering dermatomal rash is estimated to be approximately $30 \%$ to $50 \%$, with a sharp increase in incidence after the age of 50 years (4). HZ therefore provides a superb readout of age-related immunodeficiency. In the elderly, HZ is frequently complicated by persistent postherpetic neuralgic pain, even after the rash has healed, thus imposing a considerable burden on the health care system $(4,5)$. The failure to control the dormant virus in the elderly has been attributed to several age-related abnormalities in CD4 T cell biology such as a loss of virus-reactive memory $\mathrm{T}$ cells, a decline in the diversity of VZV-specific CD4 T cells, and defective CD4 T cell memory generation (6-8). Alternatively, age-related deficiency of anti-VZV T cell immunity may reflect functional exhaustion of virus-specific $\mathrm{CD}^{+} \mathrm{T}$ cells, which have been reported to express the immunoinhibitory receptor programmed death-1 (PD-1) (9-11). Other defects, such as age-related loss of antigen-presenting functions, have not been identified.

Authorship note: R. Watanabe and T. Shirai contributed equally to this work. Conflict of interest: The authors have declared that no conflict of interest exists. Submitted: December 7, 2016; Accepted: April 27, 2017.

Reference information: / Clin Invest. 2017;127(7):2725-2738.

https://doi.org/10.1172/JCI92167.
Like VZV reactivation, coronary artery disease (CAD) is an age-related disease, with older age representing one of the key risk factors (12-14). Notably, the risk of $\mathrm{HZ}$ attacks is significantly increased in patients with CAD when compared with age-matched controls, with comorbid diabetes mellitus (DM) adding additional risk $(15,16)$. Likewise, within 2 years of $\mathrm{HZ}$ diagnosis, the risk of cardiovascular events is clearly elevated (17). Traditionally, $\mathrm{CAD}$ and the underlying atherosclerosis have been recognized as vaso-degenerative conditions caused by the subendothelial deposition of lipids, which then elicit a maladaptive repair process (18). More recently, attention has moved to the immunopathogenesis of atherosclerosis, with both innate and adaptive immune responses contributing to the initiation and progression of disease (19-21). Monocytes, differentiating into tissue macrophages upon tissue infiltration, are critically important in lipid uptake and storage as well as foam cell formation (22). We and others have reported that patients with $\mathrm{CAD}$ have expanded populations of intermediate $\mathrm{CD} 14^{+} \mathrm{CD} 16^{+}$monocytes, which differentiate into proinflammatory effector macrophages $(23,24)$. Macrophages from CAD patients are metabolically reprogrammed and have a signature of excessive glucose uptake, increased glycolytic activity, and exuberant mitochondrial activity resulting in high levels of intracellular ROS. ROS overload modifies the glycolytic enzyme pyruvate kinase M2 (PKM2), favoring dimer formation, and enables PKM2 import into the nucleus, where the enzyme "moonlights" as a protein kinase, phosphorylates STAT3, and turns the cell into an IL-1 $\beta$ and IL-6 hyperproducer $(24,25)$. Changes in the M2 isoform of pyruvate kinase, shared by inflammatory macrophages and cancer cells, have metabolic consequences that possibly affect the cells' functional behavior. Inactivation of PKM2 leads to a decrease in lactate 
A Control PBMC

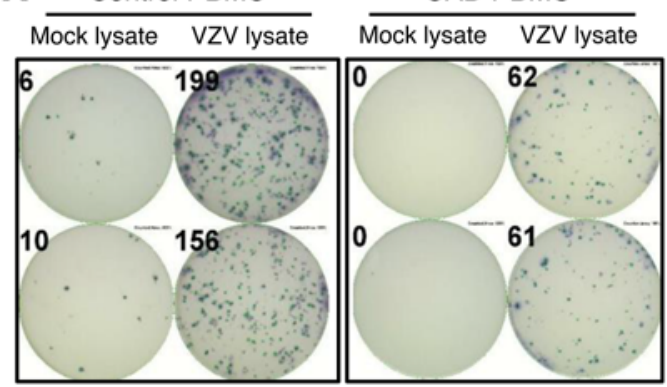

B

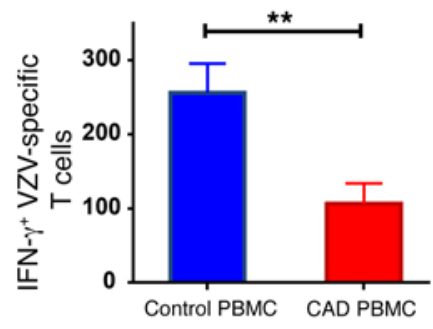

Figure 1. Protective immunity against VZV is impaired in patients with CAD. PBMCs were isolated from patients with CAD $(n=17)$ and agematched healthy controls $(n=44)$, stimulated with VZV lysate or a mock lysate for 18 hours, and plated at $1 \times 10^{6}$ cells/well. Spots of IFN- $\gamma$-secreting T cells were determined by ELISPOT assay. (A) Representative ELISPOT results. (B) The frequencies of VZV-specific IFN- $\gamma$-secreting T cells in both study cohorts are presented as the mean \pm SEM. ${ }^{*} P<0.01$, by Mann-Whitney $U$ test. production and accumulation of upstream glycolytic intermediates such as phosphoenolpyruvate. As glucose-derived carbons are diverted from glycolytic breakdown into anabolic processes, pyruvate becomes less available. Under conditions of inactivated PKM2, conversion of pyruvate into lactate slows down, and mitochondrial respiration, including mitochondrial ROS production, increases $(26,27)$. While mechanistically not entirely understood, PKM2 activation should lead to a reduction of mitochondrial pyruvate.

How metabolic reprogramming of macrophages affects effector functions beyond cytokine production is not well defined. Of interest are the functional activities through which macrophages instruct $\mathrm{T}$ cells. Monocytes and macrophages serve as antigen-presenting cells and deliver costimulatory and coinhibitory signals to interacting $\mathrm{T}$ cells (28). Macrophages specialize in antigen uptake, processing, and presentation and can fine-tune $\mathrm{T}$ cell reactivity in macrophage-rich tissue lesions such as the atherosclerotic plaque (29). By expressing immunoinhibitory ligands, such as programmed death ligand-1 (PD-L1), they can also suppress T cell immunity, a functional aspect of importance in $\mathrm{PD}-\mathrm{L}^{\mathrm{hi}}$ tumor-associated macrophages that abolish antitumor $\mathrm{T}$ cell responses (30). PD-L1 expression on cancer and tumor-associated cells has become the target of checkpoint inhibitor therapy that unleashes antitumor $\mathrm{T}$ cells and has revolutionized cancer immunotherapy (31-33).

The receptor for PD-L1 is expressed on T cells and B cells, and its ligation provides a powerful negative signal that blocks proliferation, survival, and effector functions (34-36). The density of PD-L1 on the surface of antigen-presenting cells is therefore a key element in setting the threshold for the PD-1 immune checkpoint (37). PD-L1 expression is regulated by multiple signaling pathways and transcriptional and epigenetic factors (34, 35). Both PI3K and MAPK pathways participate in controlling PD-L1 expression (38). The PDL1 promoter contains regulatory elements responsive to IFN regulatory factor 1 (IRF1), hypoxia-inducible factor $1 \alpha$ (HIF1 $\alpha$ ), and STAT3 (39-41). miRs such as miR570, miR-513, miR-34a, and miR-200 have been implicated in the negative regulation of $\mathrm{PD}-\mathrm{L} 1$ expression (34). PD-L1 is expressed on macrophages, DCs, T cells, B cells, as well as on nonlymphoid parenchymal cells; however, whether PD-L1 has a place in regulating T cell responses, and specifically VZV immunity, in patients with CAD is currently unknown.

Here, we found that monocyte-derived and tissue-infiltrating macrophages from CAD patients have immunosuppressive properties and inhibit the activation and proliferation of interacting CD4 T cells. The defect relates to the constitutive expression of PD-L1 on patients' macrophages and can be targeted by
anti-PD-L1 antibodies, which rescue the induction of antiviral T cell immunity. Remarkably, aberrant PD-L1 expression on immunosuppressive CAD macrophages, a feature shared with cancer cells, is regulated by the cells' nutrient supply. Activation of the immunoinhibitory PD-1 checkpoint represents a nutrient stress response, elicited by oversupply of the glycolytic metabolite pyruvate to the mitochondria. CAD macrophages respond to excess pyruvate by upregulating bone morphogenetic protein 4 (BMP4), which in turn activates the phosphorylated SMAD1/5/IRF1 (p-SMAD1/5/IRF1) signaling axis to induce high levels of surface PD-L1. Thus, immunosuppressive functions of CAD macrophages are under metabolic control and correctable by interfering with the mitochondrial pyruvate load. We have identified several means of undermining the aberrant PD- 1 checkpoint activation in $\mathrm{CAD}$, including smoothening glycolytic flux by forcing PKM2 into a tetrameric configuration and blocking mitochondrial pyruvate import. Shielding the mitochondria in CAD macrophages from pyruvate oversupply may enable the restoration of protective immunity, such as antiviral as well as antitumor $\mathrm{T}$ cell responses in patients with CAD.

\section{Results}

Impaired anti-VZV immunity in patients with CAD. The risk of reactivating VZV and suffering from shingles attacks increases progressively with age, but is $20 \%$ to $30 \%$ higher in patients with CAD $(15,16)$. The immune system controls chronic VZV infection through virus-specific CD4 T cells, which release IFN- $\gamma$ (3). VZV-specific $T$ cell responses can be quantified in an ex vivo system by loading antigen-presenting cells with VZV lysate and measuring the frequency of IFN- $\gamma$-releasing $\mathrm{T}$ cells in an ELISPOT assay system $(6,7)$. IFN- $\gamma$ production measured in this assay system derives almost exclusively from $\mathrm{CD} 4^{+} \mathrm{T}$ cells. In a cohort of healthy individuals aged 62-84 years, 1 of 4,000 cells responded to VZV antigen with IFN- $\gamma$ release, indicating that these healthy subjects had developed robust immune memory against the virus (Figure 1). Frequencies measured in peripheral blood mononuclear cells (PBMCs) from patients with CAD (mean age, 69.9 years) were 2.5 -fold lower, amounting to an estimated frequency of only 1 VZV-reactive cell per 10,000 PBMCs.

These data identified a defect in the antiviral $\mathrm{T}$ cell response of CAD patients and provided an explanation for the increased risk of CAD patients of suffering VZV reactivation.

CAD macrophages suppress $T$ cell activation and clonal expansion. To investigate whether the reduced $\mathrm{T}$ cell reactivity against VZV antigen resulted from deficient antigen-presenting function, 
A

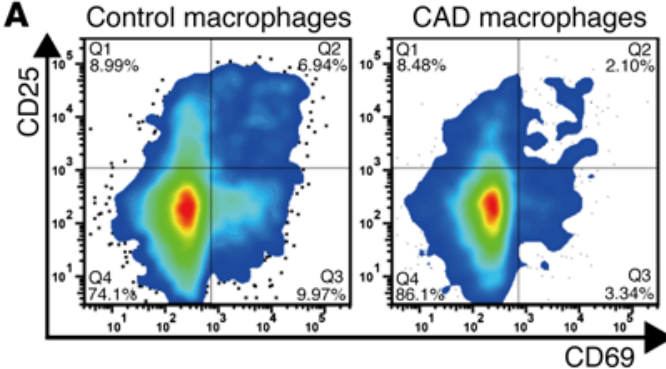

B

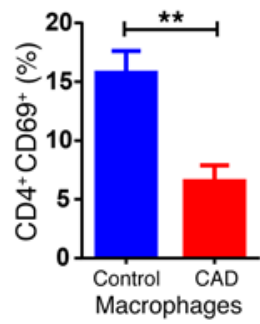

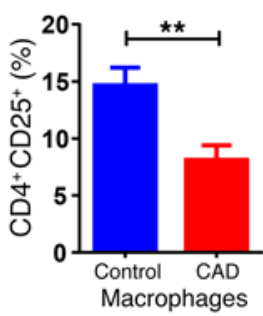

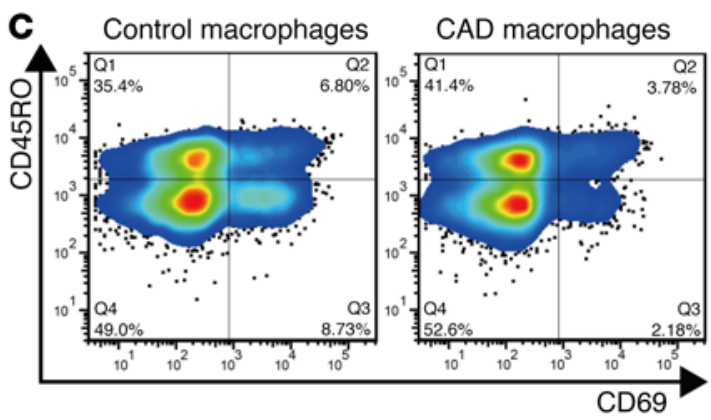

D

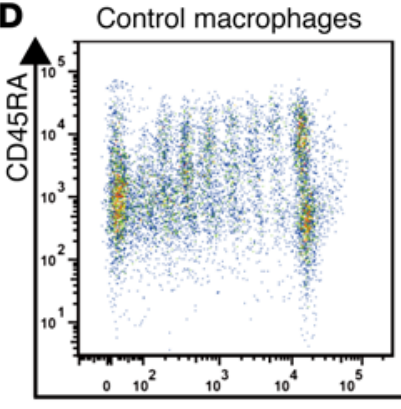

CAD macrophages

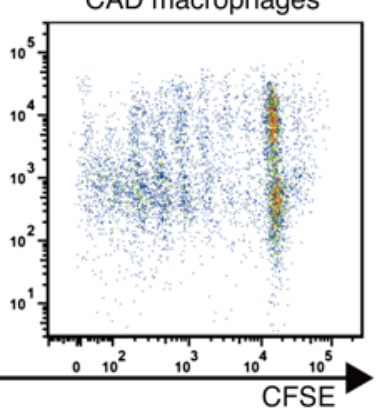

E

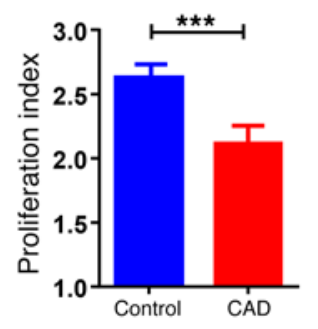

Figure 2. CAD macrophages fail to support activation and clonal expansion of CD4 T cells. Macrophages were generated from monocytes from $C A D$ patients and age-matched controls and cocultured with healthy purified CD4 T cells (macrophages: $10,000 /$ well; T cells: $50,000 /$ well). The stimulatory capacity of the macrophages was determined by the induction of CD69 and CD25 on T cells after 72 hours. T cell proliferation was quantified by CFSE dilution. (A) Representative flow cytometric data. (B) Frequencies of $\mathrm{CD4} 4^{+} \mathrm{CD} 69^{+}$and $\mathrm{CD} 4{ }^{+} \mathrm{CD} 25^{+} \mathrm{T}$ cells from 8 independent experiments. (C) Inhibitory effect of CAD macrophages on CD45RO- and CD45RO ${ }^{+} \mathrm{CD}^{+} \mathrm{T}$ cell populations. T cell activation was measured by the frequency of CD69+CD4+ $T$ cells. Representative dot blots are shown. (D-F) T cell proliferation was quantified by CFSE dilution on day 6. (D) Representative dot blots. (E) Proliferation indices for 8 healthy controls and 13 patients with CAD. (F) The deficiency of $T$ cell stimulation by CAD macrophages increased with increasing numbers of macrophages, suggestive of an active suppressive mechanism and not a reduced stimulatory capacity. Flow cytometric measurements of CFSE dilution in representative cocultures are shown. All data represent the mean \pm SEM. ${ }^{* *} P<0.01$ and ${ }^{* * *} P<0.001$, by unpaired, 2-tailed Student's $t$ test.

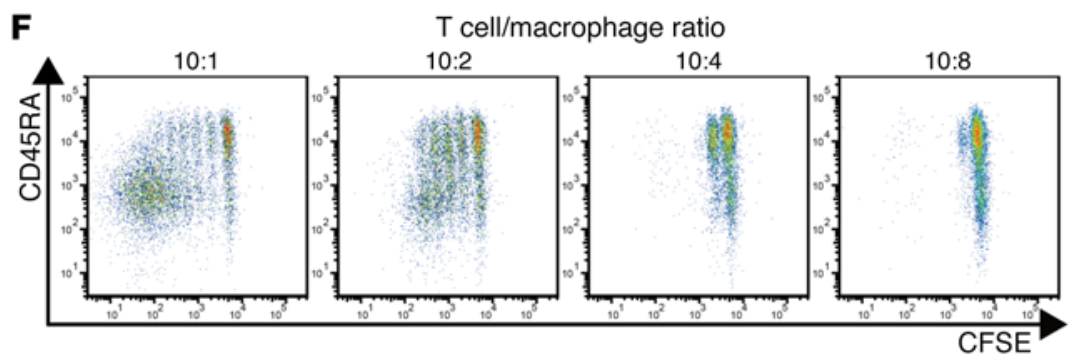

we tested the ability of patient-derived macrophages to activate healthy CD $4 \mathrm{~T}$ cells. Macrophages were generated from circulating $\mathrm{CD} 14^{+}$precursors, loaded with anti-CD3 antibodies to equalize the T cell receptor-directed signal, and cocultured with purified healthy CD4 T cells. We relied on paired samples of patient-derived and control macrophages, which were tested with identical allogeneic CD4 T cells. The ability of CAD macrophages to initiate and sustain $\mathrm{CD} 4 \mathrm{~T}$ cell stimulation was quantified by the frequency of $\mathrm{CD} 4 \mathrm{~T}$ cells upregulating the activation marker CD69 or CD25. T cell proliferative expansion was determined through dilution of the cellular dye CFSE. We observed that markedly fewer CD4 T cells entered the activation cascade (Figure 2, A and B) or proliferated (Figure 2, D and E) when they were cocultured with CAD macrophages as opposed to healthy macrophages. The frequency of $\mathrm{CD} 4^{+} \mathrm{CD} 69^{+} \mathrm{T}$ cells was 3-fold lower when the activating signals were provided by patient-derived macrophages. The inability of CAD macrophages to drive $\mathrm{CD} 4 \mathrm{~T}$ cells into the activation cycle was particularly relevant for naive $\mathrm{CD}^{2} 5 \mathrm{RO}^{-} \mathrm{T}$ cells (Figure $2 \mathrm{C}$ ), a CD4 $\mathrm{T}$ cell subpopulation that is highly dependent on costimulation. We noticed that the failure of CAD macrophages to convert naive $\mathrm{T}$ cells into memory $T$ cells and promote clonal expansion was sensitive to the number of macrophages present in the cultures (Figure 2F). With increasing numbers of macrophages, and thus decreasing $\mathrm{T}$ cell/macrophage ratios, $\mathrm{T}$ cells were essentially unable to enter the cell cycle and expand. This finding suggested an active mechanism mediating suppression, instead of a lack of costimulation.

These data identified CAD macrophages as immunoinhibitory effector cells.

Activation of the glycolytic enzyme PKM2 reverses the immunoinhibitory functions of CAD macrophages. CAD macrophages are 
A

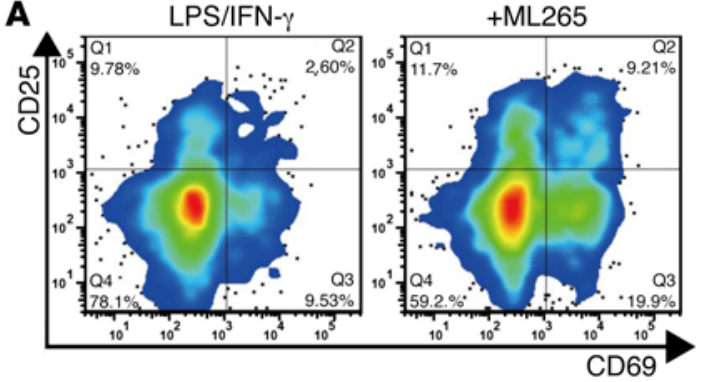

D

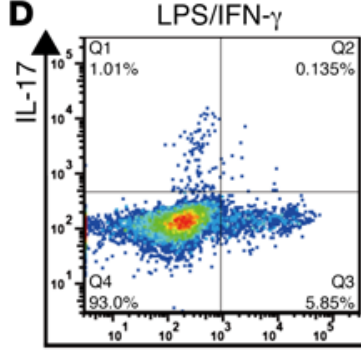

B
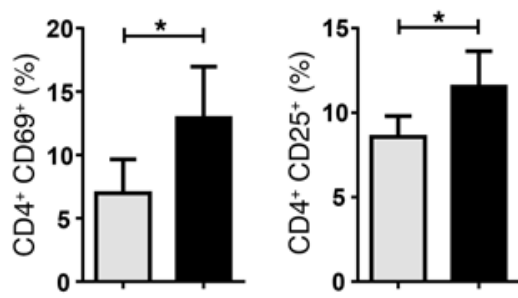

E
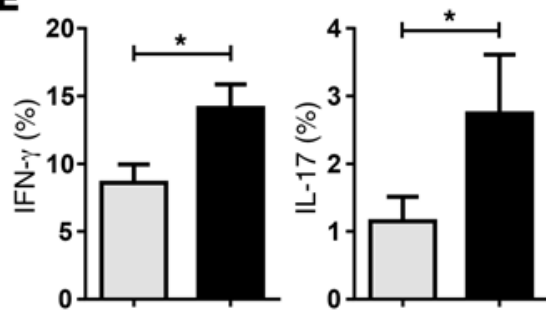

C

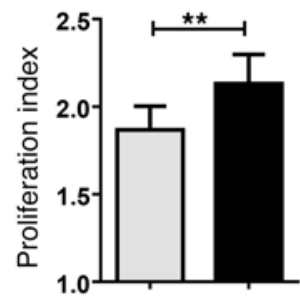

口 LPS/IFN- $\gamma$

LPS/IFN- $\gamma+$ ML265

Figure 3. Activation of PKM2 corrects the inhibitory function of CAD macrophages. Healthy CD4 T cells were cocultured with LPS/IFN- $\gamma$-stimulated macrophages generated from patients with CAD who were pretreated with vehicle or the PKM2 activator ML265 ( $50 \mu M$ ). Induction of the T cell activation markers CD25 and CD69 was measured by flow cytometry after 72 hours. (A) Representative contour plots. (B) Frequencies of CD4 $4^{+} \mathrm{CD} 25^{+}$and $\mathrm{CD} 4{ }^{+} \mathrm{CD} 69^{+}$ T cells from 7 independent experiments. (C) Purified CD4 T cells were stained with CFSE and cocultured with vehicle or ML265-pretreated macrophages. Proliferation of CD4 T cells was analyzed by CFSE dilution on day 6. Proliferation indices are from 7 independent experiments. (D and E) Commitment of CD4 T cells to IFN- $\gamma$ or IL-17 production after coculture with vehicle or ML265-pretreated macrophages was measured by intracellular cytokine staining on day 7. (D) Representative dot plots. (E) Frequencies of CD4+IFN- $\gamma^{+}$and CD4+IL-17+ $T$ cells from 6 independent experiments. All data represent the mean \pm SEM. ${ }^{*} P<0.05$ and ${ }^{* *} P<0.01$, by paired, 2 -tailed Student's $t$ test.

metabolically reprogrammed, overutilizing glucose, which stresses the mitochondrial machinery and results in excessive ROS production. One consequence is the dimerization of the glycolytic enzyme PKM2, which promotes the production of IL- 6 and IL-1 $\beta$ and renders the macrophages hyperinflammatory (24).

To examine whether the metabolic abnormalities in CAD macrophages are linked to their antigen-presenting and $\mathrm{T}$ cell stimulatory functions, PKM2 function was modulated with the small-molecule reagent ML265, which forces PKM2 into a highly glycolytic tetrameric formation (42), offsetting PKM2 inactivation and the low-pyruvate state of the cells. Pretreatment of CAD macrophages with ML265 counteracted the suppressive function and restored effective $\mathrm{T}$ cell stimulation (Figure $3 \mathrm{~A}$ ). The frequencies of $\mathrm{CD}^{+}{ }^{+} \mathrm{CD} 69^{+}$and $\mathrm{CD} 4{ }^{+} \mathrm{CD} 25^{+} \mathrm{T}$ cells improved significantly when PKM2 activity was enhanced (Figure 3B). Also, pretreatment with ML265 had a profound impact on the proliferation of CD4 ${ }^{+}$ $\mathrm{T}$ cells and their differentiation into effector cells (Figure 3, C-E). We measured $\mathrm{T}$ cell proliferation indices by CFSE dilution (Figure 3C), which revealed a proproliferative effect when macrophage PKM2 was activated. In the presence of untreated CAD macrophages, less than $10 \%$ of CD 4 T cells differentiated into IFN- $\gamma-$ producing Th1 cells, and approximately $1 \%$ of $\mathrm{CD}^{+} \mathrm{T}$ cells committed to the Th17 lineage (Figure 3, D and E). Both effector cell populations increased by $50 \%$ when the CAD macrophages were pretreated with the PKM2 activator. We tested whether ML265 treatment causes conditioning of the extracellular environment, e.g., a reduction in glucose availability that would indirectly affect $\mathrm{T}$ cell activation and growth. In transfer experiments, in which
$\mathrm{T}$ cells were placed into medium conditioned by M265-treated macrophages, $\mathrm{T}$ cell activation was as effective as that observed in T cells kept in the control medium (Supplemental Figure 1; supplemental material available online with this article; https://doi. org/10.1172/JCI92167DS1).

These data established a functional connection between the glycolytic machinery and macrophage functions, specifically in $\mathrm{T}$ cell-macrophage interactions.

$P K M 2$ regulates $P D-L 1$ expression. To understand how the glycolytic enzyme PKM2 can modulate antigen-presenting functions of macrophages, we analyzed whether PKM2 activation influences the gene expression pattern of costimulatory and coinhibitory ligands. We focused on 4 costimulatory molecules that are critically involved in coactivating $\mathrm{CD}^{+} \mathrm{T}$ cells: the constitutively expressed CD80 and CD86 and the activation-induced ICOS ligand and OX40 ligand (43). Pretreatment of macrophages did not affect any of the costimulatory ligands (Figure 4A). Unexpectedly, we found that CAD macrophages were high expressers of the coinhibitory ligand PD-L1. PD-L1 is a critical regulator of antitumor $\mathrm{T}$ cell immunity and is the target of immunostimulatory therapy in cancer patients $(36,44)$. Upon activation, CAD macrophages increased the surface expression of CD86 by approximately 2 -fold, while PD-L1 density increased by almost 10 -fold (Figure 4, B and C). Enhancing glycolytic flux by treatment with the PKM2 modulator ML265 did not affect CD86 expression, but reduced $\mathrm{PD}-\mathrm{L} 1$ by $40 \%$.

ML265, by restoring glycolytic flux and pyruvate-to-lactate conversion, effectively reduces mitochondrial pyruvate import 
A
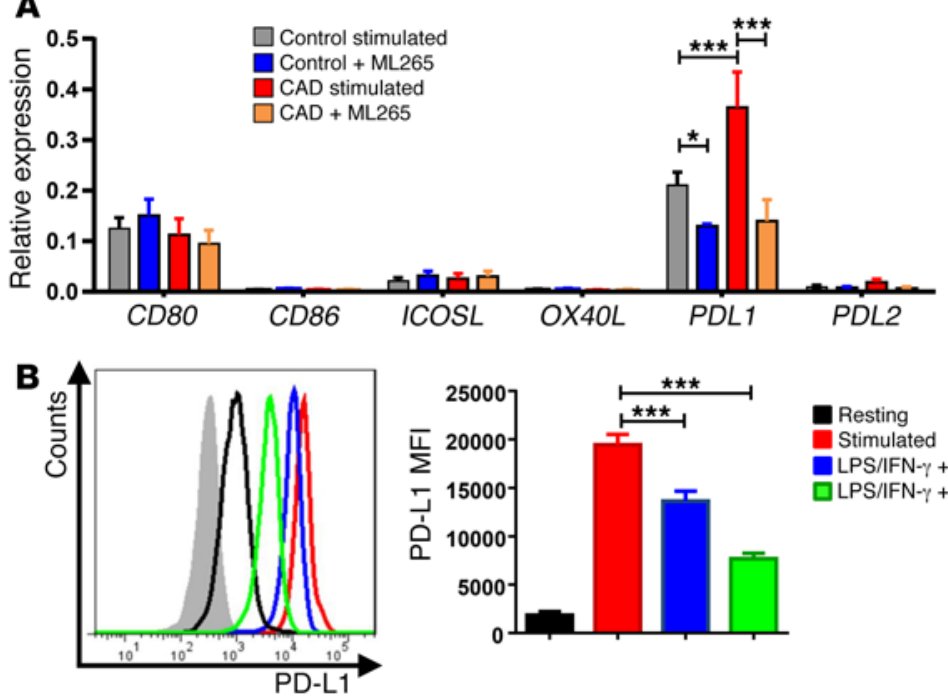

C

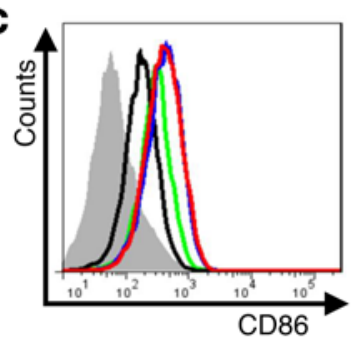

D

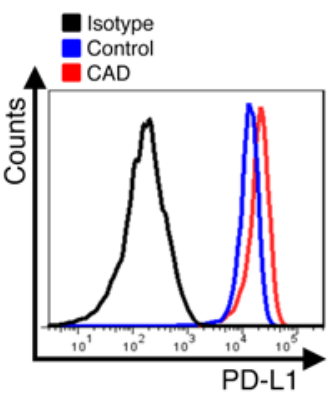

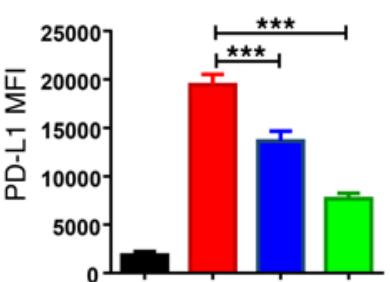

Resting

Stimulated

LPS/IFN-\% + ML265

- LPS/IFN $-\gamma+2 \mathrm{DG}$

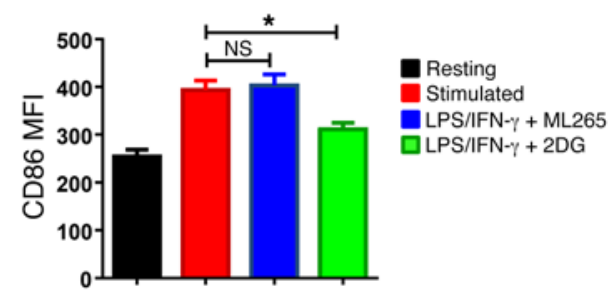

E

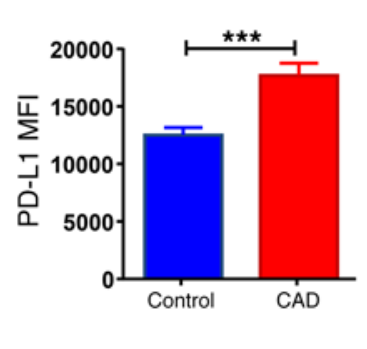

Figure 4. PKM2 regulates the expression of the immunoinhibitory ligand PD-L1. Macrophages were generated from patients with $C A D$ and age-matched healthy controls and stimulated with LPS and IFN- $\gamma$ for 24 hours. In parallel cultures, the PKM2 activator ML265 $(50 \mu \mathrm{M})$ or the glucose analog 2DC (5 mM) was added as indicated. (A) Gene expression of costimulatory or coinhibitory molecules measured after 24 hours by reverse transcription PCR (RT-PCR). Data are from 7 experiments. (B and C) Surface expression of the coinhibitory ligands PD-L1 and the costimulatory ligand CD86 was measured by flow cytometry. Representative histograms and mean florescence intensity (MFI) from 9 experiments. (D and $\mathbf{E}$ ) PD-L1 surface expression on activated macrophages from 18 healthy controls and 34 CAD patients measured by flow cytometry. (D) Representative histograms. (E) Summarized data from both study cohorts. (F) Correlation between PD-L1 surface expression on activated macrophages and the number of comorbidities (DM, HTN, HL) for 34 CAD patients. All data represent the mean \pm SEM. ${ }^{*} P<0.05$ and ${ }^{* *} P<0.001$, by 2-way ANOVA (A-C), Mann-Whitney $U$ test (E), and 1-way ANOVA (F).
$(26,27)$. Dampening of mitochondrial respiration by disrupting glycolytic breakdown can be mimicked by feeding the cells with 2-deoxy glucose (2DG). We found that inhibiting glycolysis through 2DG effectively reduced PD-L1 expression (Figure 4B) even more so than did treatment with ML265. 2DG-mediated disruption of glycolytic activity was sufficient to also reduce CD86 expression (Figure 4C), indicating that the glucose-deprived cells were under metabolic stress.

These data mechanistically connected PD-L1 expression to glycolytic flux, with PKM2-inactivated cells expressing the highest levels of PD-L1.

$C A D$ macrophages are constitutively $P D-L 1^{h i}$. We addressed the question of whether $\mathrm{CAD}$ macrophages have higher expression of the immunoinhibitory ligand PD-L1. In a cohort of 18 heathy individuals and 34 patients with CAD, we compared PD-L1 surface expression (Figure 4, D and E). We found that the surface density of PD-L1 was significantly higher on CAD macrophages. PD-L1 levels measured by flow cytometry were similarly elevated on macrophages generated from male and female patients (Sup- plemental Figure 2), excluding sex as a determining factor. Since patients with CAD frequently have comorbidities such as DM, hypertension (HTN), or hyperlipidemia (HL), we asked whether PD-L1-expressing macrophages were associated with such comorbidities. Patients were categorized on the basis of the number of comorbidities they had at the time of study enrollment (Figure 4F). PD-L1 surface expression was similar in patients with one or several comorbidities, identifying the cardiovascular disease process itself as the major determinant. To exclude the possibility that medications used routinely to treat CAD patients increase surface expression of PD-L1, we explored whether treatment with aspirin, statins, and an angiotensin-converting enzyme inhibitor affected PD-L1 expression (Supplemental Figure 2). We found that surface densities of PD-L1 were similar in all individuals, independent of their medication exposure.

$P D-L 1$ expression on macrophages residing in the atherosclerotic lesion. Next, we examined whether PD-L1 is present on tissue-residing macrophages in atherosclerotic lesions. To capture all stages of the atherosclerotic disease process, we collected aortic wall speci- 

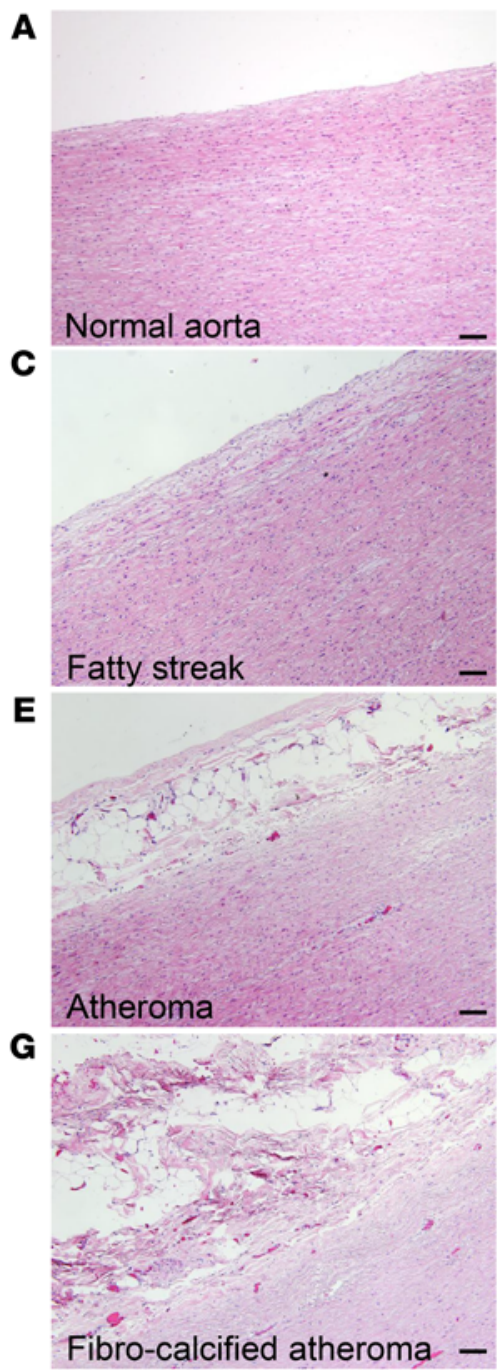
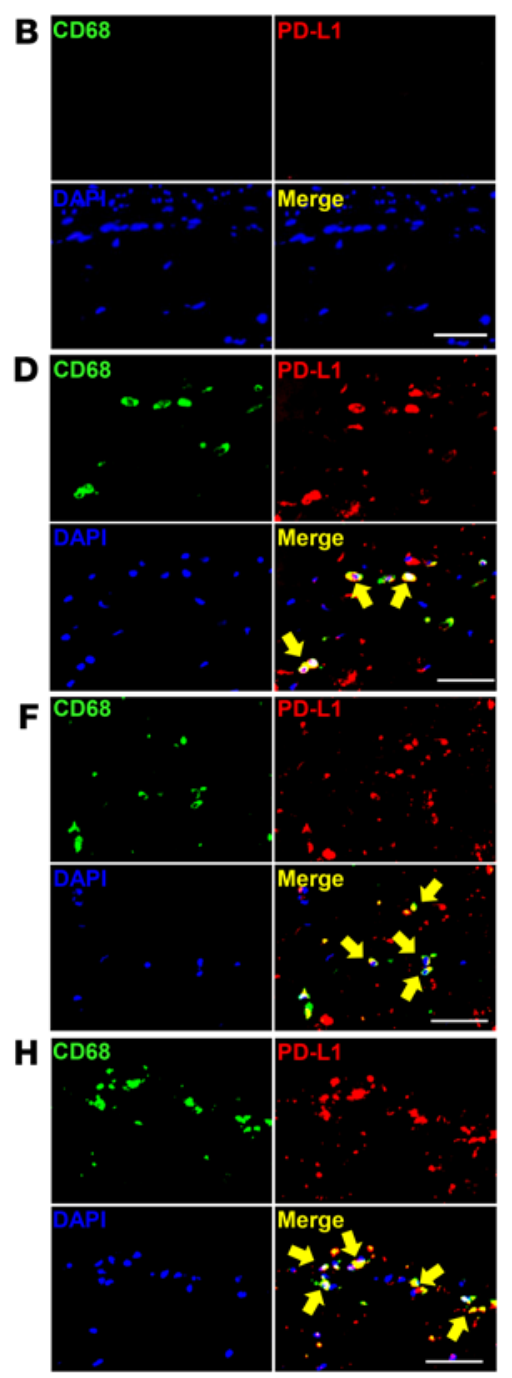

Figure 5. Tissue-resident macrophages express the immunoinhibitory ligand PD-L1 through all stages of atherosclerosis. (A, C, E, and $\mathbf{C}) \mathrm{H} \& \mathrm{E}$ staining from aortic wall sections. (A) Normal wall; (C) fatty streak; (E) atheroma; and (C) fibrocalcified atheroma. Scale bars: $20 \mu \mathrm{m}$. (B, D, F, and H) PD-L1-expressing macrophages identified by triple-color IHC in normal wall (B), fatty streak (D), atheroma (F), and fibrocalcified atheroma $(\mathbf{H})$ from aortic wall sections. Tissue sections were immunostained with anti-CD68 (green), antiPD-L1 (red), and DAPI (blue) and analyzed by fluorescence microscopy. Scale bars: $20 \mu \mathrm{m}$. Images from 1 of 6 stains are shown. Yellow arrows mark macrophages expressing PD-L1. mens with different atherosclerotic lesions and analyzed them by triple-color IHC (Figure 5). We found that normal aortic wall was essentially free of macrophages. Fatty streak lesions, representing early lesions, contained $\mathrm{CD} 68^{+}$macrophages, the majority of which stained positive for PD-L1 (Figure 5, C and D). Macrophages within fully developed atheromas, as well as those in fibrocalcified lesions, were consistently PD-L1 ${ }^{+}$, establishing the immunoinhibitory ligand as a common feature of plaque-infiltrating macrophages.

Collectively, tissue-resident macrophages in patients with CAD are primarily immunosuppressive because of high expression of the inhibitory ligand PD-L1.

Macrophage-mediated $T$ cell suppression is $P D-L 1$ dependent. To confirm the role of PD-L1 in CAD macrophages, we applied siRNA technology to reduce the surface expression of the ligand in patient-derived macrophages. PD-L1 siRNA transfection resulted in a marked downregulation of surface PD-L1 expression, but PD-L2 expression was not affected (Supplemental Figure 3). When PD-L1 was knocked down in CAD macrophages, the ability to activate $\mathrm{CD} 4 \mathrm{~T}$ cells, measured by the frequencies of $\mathrm{CD} 4{ }^{+} \mathrm{CD} 25^{+}$and $\mathrm{CD}^{+}{ }^{+} \mathrm{CD} 69^{+} \mathrm{T}$ cells, improved significantly (Figure 6, A and B), and the capacity to induce $\mathrm{T}$ cell proliferation, measured by CFSE dilution, was also restored (Figure 6, C and D).
We next examined whether blocking access to PD-L1 counteracts the suppressive capabilities of CAD macrophages. Blocking PD-L1 using anti-PD-L1 antibodies effectively restored the T cellstimulatory functions of CAD macrophages (Figure 6, E-H). When anti-PD-L1 antibodies were combined with the PKM2 activator ML265, we observed no further improvement of T cell activation, suggesting that the effect of ML265 on repairing stimulatory macrophage functions was mediated by PD-L1 downregulation.

To confirm that PD-L1 overexpression was functionally important in impairing the patients' antiviral immunity, we blocked the immune checkpoint with an anti-PD-L1 antibody and assessed the impact on the frequencies of VZV-specific T cells in the ELISPOT assay system (Figure 7). We found that blocking PD-L1 improved the frequencies of anti-VZV-responsive T cells by $20 \%$ to $30 \%$ in all patients tested.

These data implicated PD-L1 as a key molecule in rendering CAD macrophages immunosuppressive.

The BMP4/p-SMAD1/5/IRF1 signaling pathway regulates $P D-L 1$ in CAD macrophages. To understand why macrophages in CAD patients are constitutively $\mathrm{PD}-\mathrm{L} 1^{\text {hi }}$, thus providing strong inhibitory signals to interacting $\mathrm{T}$ cells, we screened signaling pathway inhibitors for their ability to reduce the surface density 
A
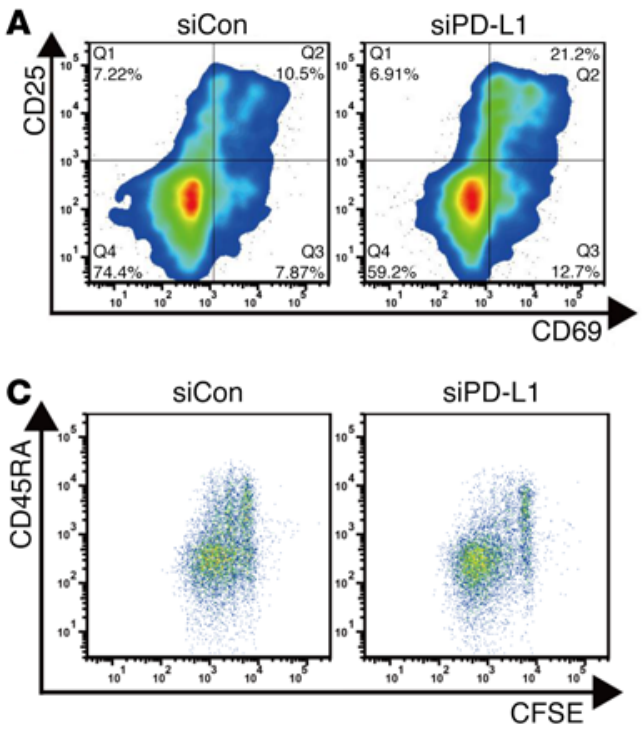

E

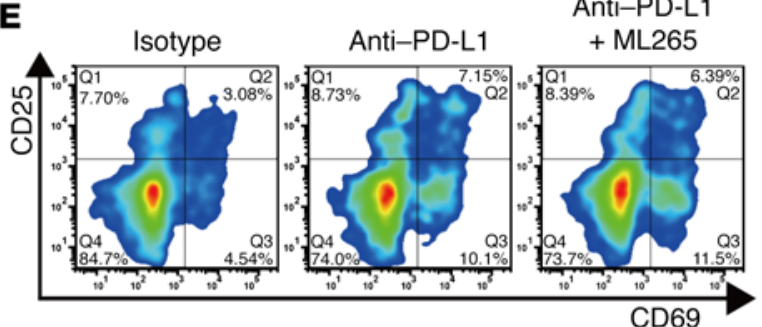

G

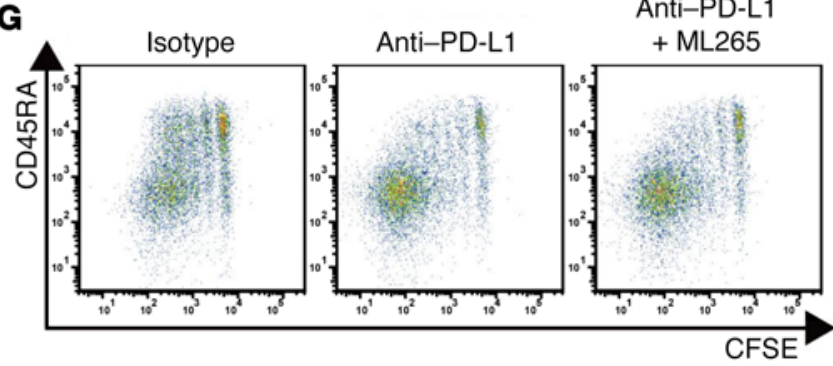

D

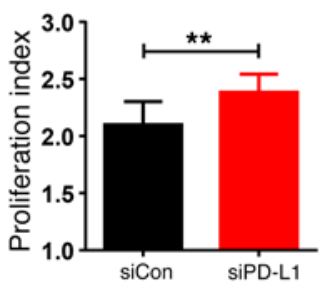

B

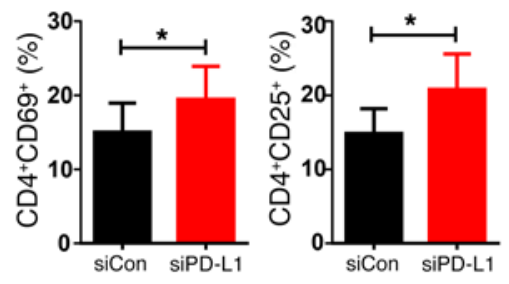

(n)
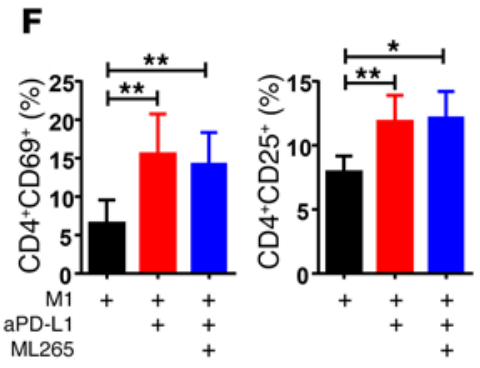

H

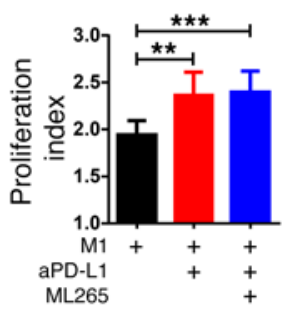

Figure 6. T cell-inhibitory functions of CAD macrophages are mediated by PD-L1. (A-H) Macrophages were generated from CAD patients and cocultured with healthy CD4 T cells. Macrophages were transfected with siRNA specific for PD-L1 (siPD-L1) or control siRNA (siCon). Alternatively, macrophages were incubated with anti-PD-L1 antibodies or an isotype control antibody. In some experiments, the PKM2 activator ML265 $(50 \mu \mathrm{M})$ was combined with antiPD-L1 antibodies. Frequencies of activated $\mathrm{CD} 4{ }^{+} \mathrm{CD} 69^{+}$and $\mathrm{CD} 4{ }^{+} \mathrm{CD} 25^{+}$ T cells were measured by flow cytometry on day 3. T cell proliferation was assessed by CFSE dilution on day 6 . (A) Representative flow cytometric contour plots for $\mathrm{CD}_{6} 9^{+}$and $\mathrm{CD} 25^{+}$ CD4 T cells. (B) Summary of 8 experiments comparing CAD macrophages transfected with either control siRNA or PD-L1-specific siRNA. (C) Representative dot plot of CFSE expression in CD4 T cells captured on day 6. (D) Summarized proliferation indices from 7 experiments are shown. (E-H) The PKM2 activator ML265 was combined with anti-PD-L1 antibodies (aPD-L1). ( $E$ and $\mathbf{F}$ ) T cell activation was measured on day 3 according to the frequency of $\mathrm{CD} 69^{+}$or $\mathrm{CD} 25^{+} \mathrm{CD} 4$ $T$ cells. Representative contour plots and results from 5 to 6 independent experiments are shown. (G and $\mathbf{H})$ Dilution of CFSE in proliferating CD4 T cells measured by flow cytometry. Representative dot plots and summarized proliferation indices from 7 experiments are shown. Values represent the mean \pm SEM. ${ }^{*} P<0.05$, ${ }^{*} P<0.01$, and ${ }^{* *} P<0.001$, by paired, 2-tailed Student's $t$ test. of PD-L1. Screening experiments revealed that treatment with the small molecule dorsomorphin at a dose of $2 \mu \mathrm{M}$ effectively lowered PD-L1 expression levels (Figure 8, A and B). A major effect of dorsomorphin lies in the dose-dependent inhibition of SMAD1/5/8 phosphorylation, inhibiting IRF1-dependent gene transcription (45). This signaling pathway is triggered by the bone morphogenetic proteins, specifically BMP4 $(46,47)$. Consequently, we investigated the impact of dorsomorphin on p-SMAD1/5 and IRF1 in CAD macrophages (Figure 8, C-E). Dorsomorphin potently reduced the concentrations of $\mathrm{p}-\mathrm{SMAD} 1 / 5$ and IRF1 in patient-derived cells. This prompted experiments comparing control and CAD macrophages for the spontaneous expression of the signaling molecule p-SMAD1/5, the transcription factor IRF1, and the upstream inducer BMP4 (Figure 8, F-H). Following activation with IFN- $\gamma$ and LPS, patient-derived macrophages had significantly higher amounts of phosphorylated SMAD1/5, and intracellular concentrations of IRF1 were similarly increased. Transcript levels of $B M P 4$ were 2.5-fold higher in CAD macrophages than in control macrophages. Increased expression of BMP4 and IRF1 transcripts and elevated surface expression of PD-L1 was already detectable in $\mathrm{CD} 14^{+}$monocytes freshly isolated from peripheral blood (Supplemental Figure 4). Also, recombinant BMP4 proved to upregulate intracellular IRF1 and directly increased the surface expression of PD-L1 in healthy macrophages (Supplemental Figure 5).

To test whether BMP4 and IRF1 expression was regulated by glycolytic flux, we treated CAD macrophages with ML265, analogous to the experiments depicted in Figure 4A. By enforcing PKM2 tetramerization, ML265 enhances flux through the glycolytic pathway and effectively reduces the availability of pyruvate for mitochondrial respiration $(26,27)$. ML265 treatment reduced BMP4 transcript levels to approximately one-third of the original levels (Figure 8I), and IRF1 protein concentrations declined significantly (Figure 8, J and K).

These data placed the macrophage product BMP4 and the transcription factor IRF1 upstream of PD-L1 induction. 
A Isotype antibody

Mock lysate VZV lysate
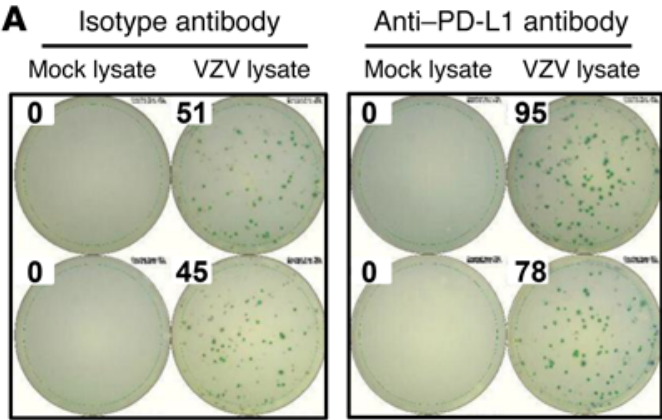

B

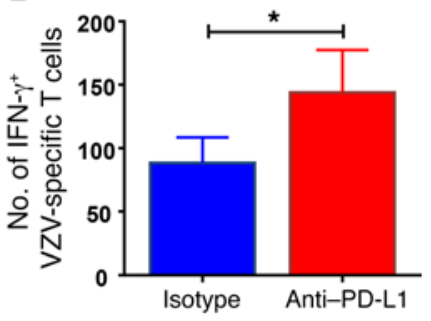

Figure 7. Blocking PD-L1 corrects VZV response in patients with CAD. PBMCs were isolated from patients with $\operatorname{CAD}(n=11)$, plated at $1 \times 10^{6}$ cells $/$ well, and stimulated with VZV lysate or a mock lysate for 18 hours in the presence of isotype control or antiPD-L1 antibody. Spots of IFN- $\gamma$-secreting T cells were determined by ELISPOT assay. (A) Representative ELISPOT results. (B) The numbers of VZV-specific IFN- $\gamma$-secreting $T$ cells are presented as the mean \pm SEM. ${ }^{*} P<0.05$, by paired, 2 -tailed Student's $t$ test.
The glycolytic intermediate pyruvate controls BMP4 and PD-L1 expression in CAD macrophages. To implicate BMP4 in the induction and sustainment of PD-L1 in vivo, tissue sections for carotid atheroma were immunostained for BMP4 protein. The protein localized exclusively to $\mathrm{CD}^{2} 8^{+}$cells, with most tissue macrophages producing BMP4 (Figure 9A).

To better understand how glycolytic activity affects BMP4 and IRF1 production, we investigated whether the critical glycolytic intermediate pyruvate influences the activity of the BMP4/IRF1/ PD-L1 axis. Healthy macrophages fed with exogenous pyruvate responded with a doubling of BMP4 transcript levels (Figure 9B). Likewise, cellular pyruvate concentrations regulated IRF1 transcription (Figure 9C). Supplying exogenous pyruvate was sufficient to increase PD-L1 surface expression (Figure 9, D and E). In essence, pyruvate-treated healthy macrophages acquired the PD-L1 ${ }^{\text {hi }}$ phenotype spontaneously encountered in CAD macrophages (Figure 9E).

To examine whether disrupting the supply of pyruvate to the mitochondria can change PD-L1 expression, we stimulated healthy and patient-derived macrophages in the presence of the pyruvate transport inhibitor UK5099 (Figure 9, F and G). CAD macrophages normalized PD-L1 surface expression when the import of pyruvate into the mitochondria was impaired. Similarly, PD-L1 expression on control macrophages was sensitive to the amount of pyruvate shuttled from the cytoplasm to the mitochondria.

These data mechanistically linked expression of the immunoinhibitory ligand PD-L1 to the availability of mitochondrial pyruvate.

\section{Discussion}

Cell-intrinsic metabolic dysregulation renders macrophages hyperinflammatory in patients with CAD. Here, we have linked nutrient excess in CAD macrophages to defects in adaptive immunity, emphasizing the interdependence of metabolic and immune regulation. Because of high surface expression of the immunoinhibitory ligand PD-L1, CAD macrophages provide negative signals to $\mathrm{T}$ cells and inhibit their activation and clonal expansion, which weakens $\mathrm{T}$ cell immunity as specifically shown for VZV. PD-L1 ${ }^{\text {hi }}$ immunosuppressive macrophages populate the atherosclerotic plaque already during early stages of the disease process. Unexpectedly, PD-L1 expression is mechanistically linked to the loading of mitochondria with the glycolytic metabolite pyruvate, implicating glucose availability in the regulation of immune effector functions. In response to excess pyruvate availability, CAD macrophages begin to produce BMP4 and trigger the p-SMAD1/5/ IRF1 signaling cascade. The end result is a PD- $\mathrm{L} 1^{\text {hi }}$-expressing macrophage, placing glucose utilization upstream of immune checkpoint regulation (Figure 10) and emphasizing the negative impact of nutrient oversupply on immune cells. Glucose-dependent reprogramming of macrophage effector functions renders CAD patients susceptible to VZV reactivation and defines novel interception points in the effort to strengthen the immunocompetence of such patients.

Macrophages from patients with CAD are functionally distinct from those of age-matched healthy individuals. The current data add $\mathrm{T}$ cell inhibition to the previously described inflammatory propensity of patient-derived cells (24). Notably, both defects, the provision of immunoinhibitory signals and the excessive release of proinflammatory cytokines, can be traced back to a common molecular root, that of the dysregulation of the glycolytic machinery. Both defects respond to the identical intervention, namely the treatment with ML265, a small-molecule activator of PKM2. In previous work (24), we have linked the release of the proinflammatory cytokines IL-1 $\beta$ and IL- 6 to the dimerization and nuclear translocation of PKM2. Nuclear accumulation of dimeric PKM2 results from ROS-mediated oxidation of the enzyme and reflects the unopposed production of mitochondrial ROS, which is in itself a consequence of excess glucose utilization, imposing mitochondrial stress responses (24). The data presented here support the notion that activation of PKM2 has additional effects beyond controlling nuclear PKM2 levels, such as reducing mitochondrial stress by lowering pyruvate availability. Under conditions of high glycolytic activity, when cellular levels of the PKM2 activator fructose 1,6-bisphosphate (FBP) are high, respiration is partially suppressed, and pyruvate is promptly converted into lactate. In contrast, when PKM2 is partially inactivated, most prominently so in cancer cells and inflammatory macrophages $(24,48-50)$, upstream glycolytic intermediates accumulate and fuel synthetic pathways, such as the pentose phosphate pathway (PPP), thereby enabling cellular growth and expansion. Although the mechanism is not understood, $\mathrm{PKM} 2^{\mathrm{lo}}$ activity also makes pyruvate available to the mitochondria, fostering oxidative phosphorylation and TCA cycle activity. Treatment with ML265 phenocopied the effects achieved with 2DG or blocking mitochondrial pyruvate import, which was compatible with the model showing that ML265 essentially reduces the pyruvate load for the mitochondria. These key findings of the current study are summarized in Figure 10.

Both macrophage defects, i.e., cytokine production and PD-L1 upregulation, already existed in circulating monocytes (Supplemental Figure 4), eliminating macrophage ex vivo generation or the tissue microenvironment as causative elements. Rather, the immune defects of CAD macrophages are strongly reminiscent 


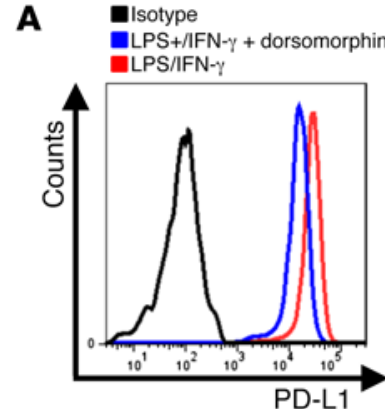

B

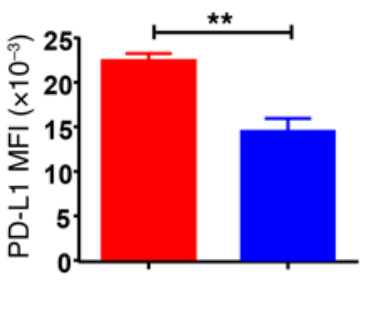

D $\quad$ Isotype

E

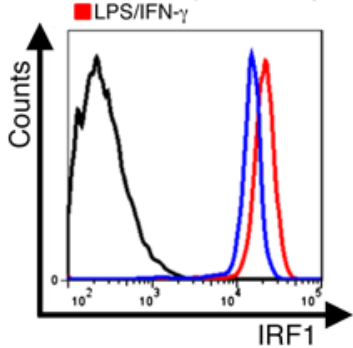

$\mathbf{F}$

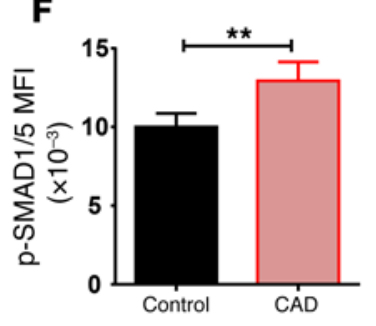

G

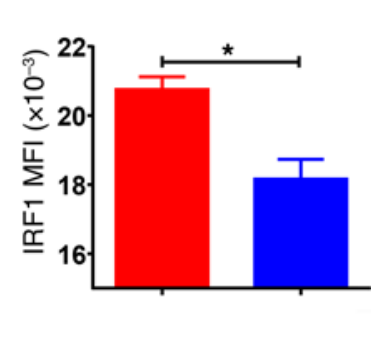

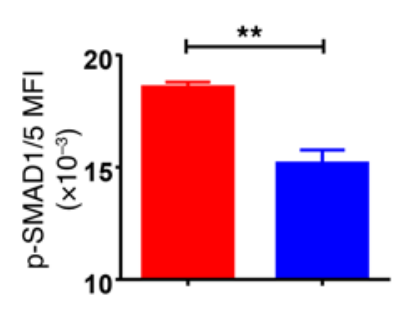

C

LPS/IFN $-\gamma$

LPS/IFN- $\gamma+$ dorsomorphin
G

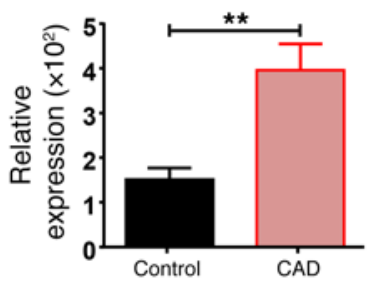

H

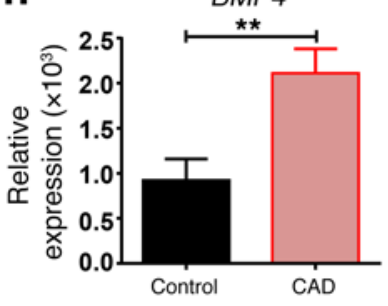

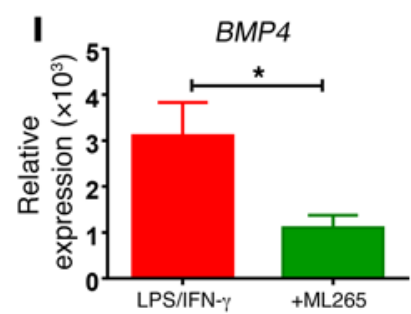

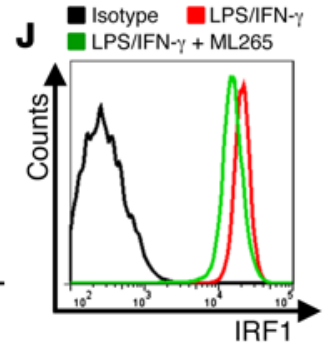

$\mathbf{K}$

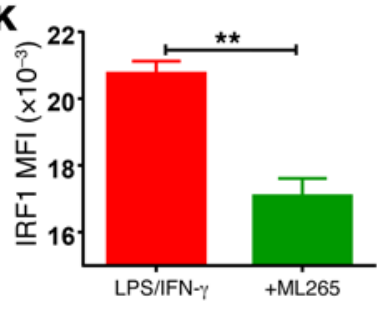

Figure 8. The BMP4/IRF1 axis drives PD-L1 expression in CAD macrophages. (A-E) Macrophages from patients with CAD were stimulated for 24 hours with LPS and IFN- $\gamma$ in the absence or presence of dorsomorphin (2 $\mu \mathrm{M})$. Flow cytometric measurement of PD-L1 surface expression (A and $\mathbf{B})$, intracellular p-SMAD1/5 (C), and IRF1 ( $\mathbf{D}$ and $\mathbf{E}$ ). Data are from 6 experiments. (F-H) Macrophages from healthy individuals and CAD patients were stimulated with LPS and IFN- $\gamma$ for 24 hours. (F) Intracellular p-SMAD1/5 levels were analyzed by flow cytometry in 6 experiments. ( $\mathbf{G}$ and $\mathbf{H}$ ) Gene expression of IRF1 (G) and BMP4 (H) was measured by RT-PCR in 9 control patient pairs. (I-K) The effect of ML265 on the induction of BMP4 transcripts (I) and expression of IRF1 protein (J and $\mathbf{K}$ ) in CAD macrophages was measured in 6 experiments, using RT-PCR and flow cytometry, respectively. All data represent the mean \pm SEM. ${ }^{*} P<0.05$ and ${ }^{*} P<0.01$, by paired 2-tailed Student's $t$ test (B, C, E, I, and $\mathbf{K})$ and Mann-Whitney $U$ test $(\mathbf{F}-\mathbf{H})$. of inflammaging, which describes the coexistence of insufficient adaptive immunity with uncontrolled tissue inflammation in aging $(51,52)$. We believe that identifying the mitochondrial pyruvate load as a molecular target underlying both abnormalities opens novel therapeutic avenues.

The key position of PD-L1 in antitumor immunity escape mechanisms has peaked interest in how this ligand is induced on immune and nonimmune cells. Inflammatory activity in the tumor microenvironment is believed to be a central driver, and the transcription factor IRF1 is primarily responsible for constitutive and IFN- $\gamma$-mediated PD-L1 expression (39). The data presented here concur with the role of IRF1 in stimulating the PDL1 promoter. However, we have implicated a different upstream inducer, BMP4. Circulating monocytes and plaque-residing macrophages produce $\mathrm{BMP} 4$, empowering them to upregulate $\mathrm{PD}-\mathrm{L} 1$ through cell-intrinsic control. The majority of plaque-infiltrating macrophages expressed both BMP4 and PD-L1, enabling them to deliver negative signals to interacting $\mathrm{T}$ cells. This mechanism may represent a failed attempt to downregulate plaque immune responses but may also undermine host-beneficial immunity, such as tissue healing (53).

BMPs are best known as regulators of bone and cartilage formation (54), but BMP4 has been implicated in modifying disease mechanisms in several cardiovascular conditions, including atherosclerosis, hypertension, valvar abnormalities, pulmonary arterial hypertension, and myocardial ischemic injuries (55-60). BMP4 is upregulated in atherosclerotic blood vessels, contributes to vascular calcification, and has been implicated in the progression of the atherosclerotic lesion (61). In an endothelial cell line, glucose increased BMP4 concentrations and ROS release in a dose-dependent manner (62). In diabetic Apoe ${ }^{-/-}$mice, BMP4 was increased in the aorta and enhanced oxidized LDL uptake in macrophages (63). BMP4 has been implicated in foam cell formation through SMAD $1 / 5 / 8$ signaling (64) and is believed to amplify cancer metastasis 

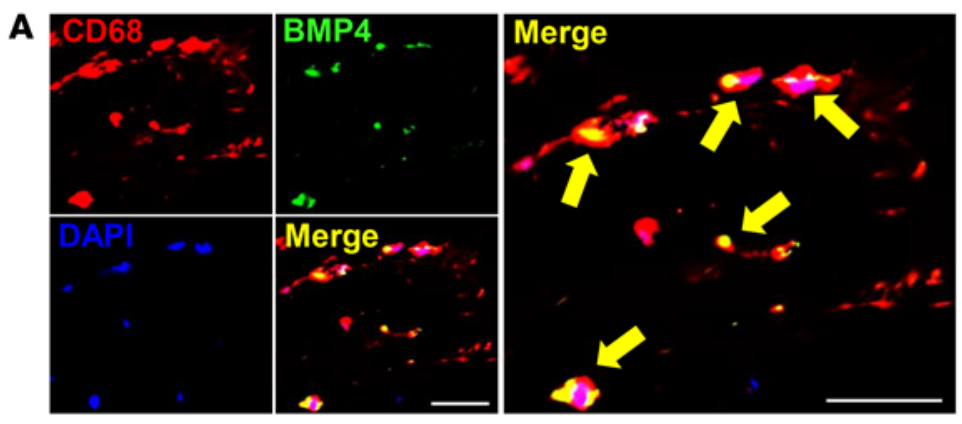

$\mathbf{B}$

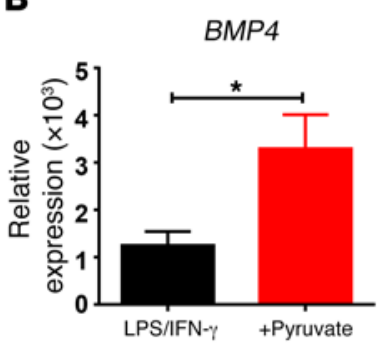

C
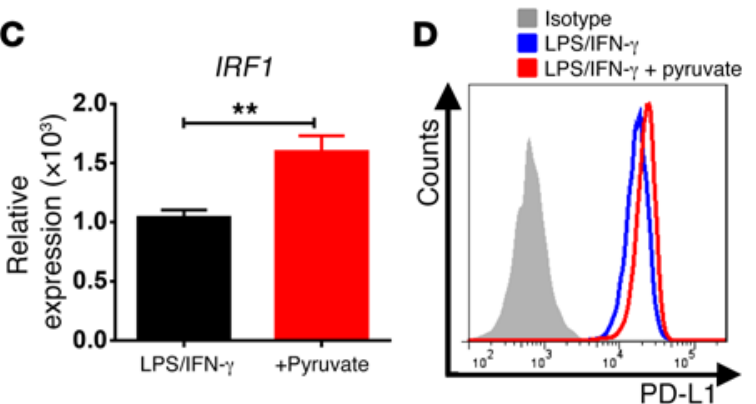

E

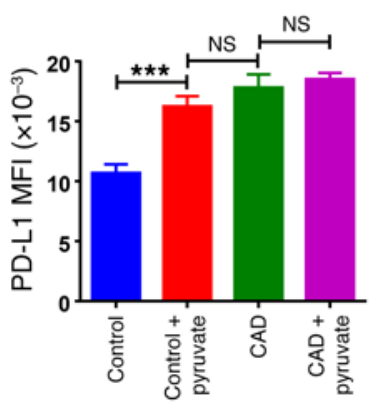

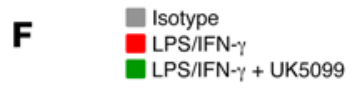

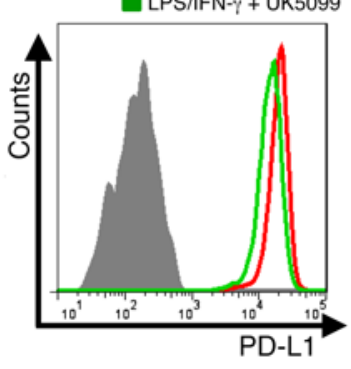

G

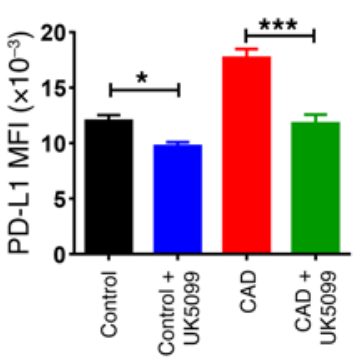

Figure 9. Pyruvate controls PD-L1 expression via the BMP4/IRF1 axis. (A) Frozen sections of carotid plaque were immunostained with anti-CD68 (red), anti-BMP4 (green), and DAPI (blue) and analyzed by fluorescence microscopy. Yellow arrows indicate BMP4-expressing macrophages. Scale bars: $200 \mu \mathrm{m}$. (B-G) Macrophages from healthy individuals and patients with CAD were stimulated with LPS and IFN- $\gamma$. As indicated, exogenous pyruvate ( $5 \mathrm{mM}$ ) was added. Alternatively, the cells were treated with the pyruvate transport inhibitor UK5099 $(2 \mu \mathrm{M})$. (B-E) Excess pyruvate upregulates BMP4, IRF1, and PD-L1 in healthy macrophages. BMP4 and IRF1 transcript levels were measured by RT-PCR (B and C), and PD-L1 surface expression was assessed by flow cytometry (D and E). Results are from 6 experiments. (F and $\mathbf{G}$ ) Inhibition of pyruvate transport into the mitochondria reduced PD-L1 expression. Macrophages from healthy individuals and patients with CAD were treated with the pyruvate transporter inhibitor UK5099 ( $2 \mu \mathrm{M})$. Surface PD-L1 expression was analyzed by flow cytometry. Results are from 7 experiments. All data represent the mean \pm SEM. ${ }^{*} P<0.05,{ }^{* *} P<0.01$, and ${ }^{* * *} P<0.001$, by paired, 2-tailed Student's $t$ test (B and $\mathbf{C}$ ) and 2-way ANOVA (E and G). and progression (65). The BMP4/p-SMAD1/5/8/IRF1 signaling network needs to be added to the pathways shaping the inflammatory environment in cardiovascular disease. The connection to the bioenergetic status of the cell is particularly appealing, as it provides a framework for integrating the metabolic abnormalities of cardiovascular disease with immune abnormalities.

Like all translational studies, the current study has limitations imposed by working with human samples. Patients were stringently selected for a high load of CAD, and we consistently matched for age and sample handling and explored the possible effects of the standard medications used to manage patients after myocardial infarction. Macrophages from non-CAD individuals treated with such medications were functionally indistinguishable from those from patients who were medication free.

We believe that this study has direct clinical implications. Recognizing that macrophages in patients with CAD are not only powerful proinflammatory effector cells, but modify T cell-dependent immune responses by providing negative signals, expands the pathogenic potential of these macrophages. Most significantly, the propensity of patients with CAD to mount hyperactive inflammatory responses is combined with impaired antiviral immunity. Reactivation of VZV is a feared complication of the immune aging process, but in patients with $\mathrm{CAD}$, disproportional impairment of
anti-VZV immunity adds further disease burden beyond vascular events. Excess PD-L1 expression is a well-recognized mechanism through which tumor cells evade $\mathrm{T}$ cell recognition and destruction, raising the question of whether $\mathrm{CAD}$ and cancer have overlapping predisposing immune defects. The risk for stroke is increased multifold in patients with lung, pancreatic, colorectal, or breast cancer (66). Also, mortality in patients with established CAD is more frequent because of noncardiovascular causes, specifically cancer, pneumonia, and sepsis (67), which is suggestive of failing immune protection in this patient population. Immunomodulatory therapy for CAD may therefore not only target the inflammatory propensity of macrophages, but may also improve their protective functions as antigen-presenting cells. Combined pro- and anti-immune functions is exemplified in tumor-associated macrophages and related myeloid cell populations (68). Unleashing antitumor $\mathrm{T}$ cells by directly blocking negative signaling with anti-PD-1 or anti-PD-L1 antibodies has caused a paradigm shift in cancer immunotherapy. The data presented here would predict that blocking the PD-1 checkpoint in patients with CAD should enhance their anti-VZV $\mathrm{T}$ cell responses in a similar manner. The sharing of inappropriate immune checkpoint activation in CAD and cancer is intriguing and may provide a rationale for the development of shared biomarkers and shared therapeutic interventions. 


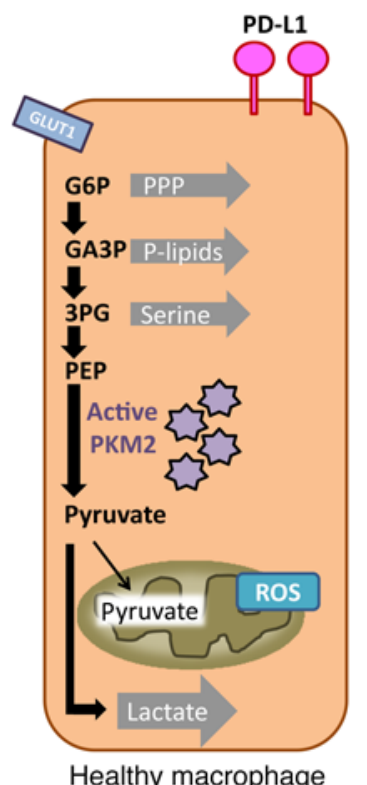

Healthy macrophage

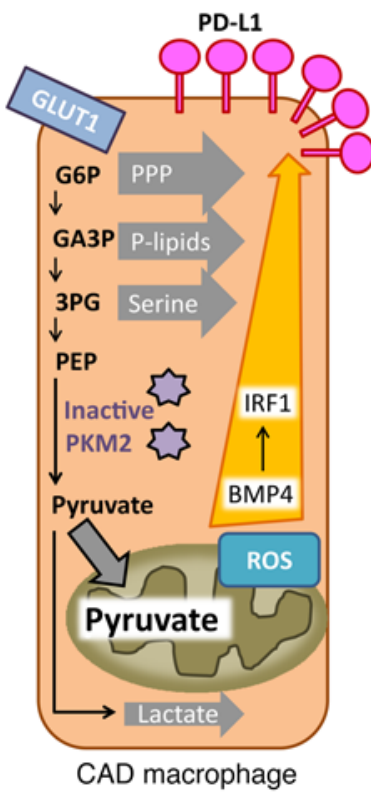

CAD macrophage

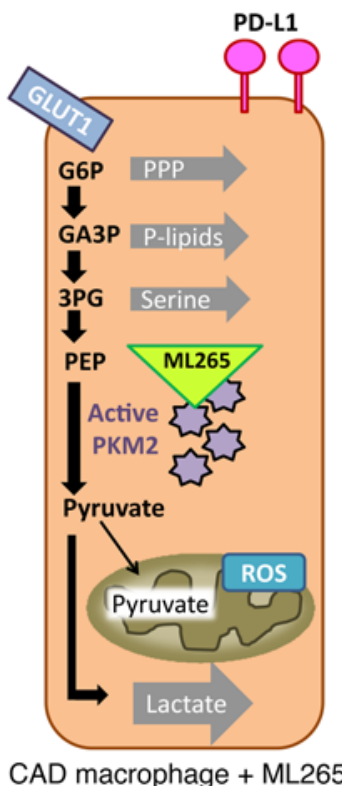

CAD macrophage + ML265
Figure 10. Proposed model connecting the pyruvate metabolic checkpoint with PD-L1 expression. In healthy macrophages (left), PKM2 is in active tetrameric form. Glycolytic flux is high, but pyruvate is mostly processed into lactate, lowering mitochondrial respiration. Macrophages from patients with CAD (middle) dimerize PKM2, lowering its activity and effectively reducing glycolytic flux and lactate production. Reduced glycolytic flux leads to the accumulation of glycolytic intermediates such as glucose 6-phosphate (G6P), glyceraldehyde 3-phosphate (GA3P), and 3-phosphoglycerate (3PG), which are used for biosynthetic purposes. Enhanced transport of pyruvate into the mitochondria causes mitochondrial stress and excess ROS production. Mitochondrial pyruvate sustains the production of BMP4 and downstream activation of the transcription factor IRF1, thereby upregulating surface expression of PD-L1. ML265 forces PKM2 into active tetrameric form (right), restores glycolytic flux, and promotes the interconversion of pyruvate into lactate. This reduces pyruvate transport into the mitochondria, eases mitochondrial stress, and prevents BMP4 induction. The end result is a normalization of PD-L1 expression. PEP, phosphoenolpyruvate; P-lipids, phospholipids.

Cells and cultures. PBMCs were isolated by density gradient centrifugation using Lymphoprep (STEMCELL Technologies). Monocytes isolated from PBMCs by plastic adherence were differentiated into macrophages in RPMI 1640 medium (Life Technologies, Thermo Fisher Scientific) supplemented with 10\% FBS (Lonza) and $20 \mathrm{ng} / \mathrm{ml}$ macrophage-CSF (M-CSF) (eBioscience) for 5 days as previously reported (24). Macrophages were further differentiated into M1 macrophages by stimulation with $100 \mathrm{U} / \mathrm{ml}$ IFN- $\gamma$ (Sino Biologicals) and $100 \mathrm{ng} / \mathrm{ml}$ LPS (Sigma-Aldrich). Macrophages were detached from plates using StemPro Accutase Cell Dissociation Reagent (Life technologies, Thermo Fisher Scientific). Total or naive $\mathrm{CD}^{+} \mathrm{T}$ cells were isolated using an EasySep Human CD4 ${ }^{+}$ T Cell Enrichment Kit or an EasySep Human Naive $\mathrm{CD}^{+}{ }^{+} \mathrm{T}$ Cell Isolation Kit (STEMCELL Technologies). Dynabeads Human T-Activator CD3/CD28 for $\mathrm{T}$ cell Expansion and Activation (Thermo Fisher Scientific; 11131D) were mixed with naive $\mathrm{CD} 4^{+} \mathrm{T}$ cells at a ratio of 1:4.

To enforce PKM2 tetramerization, $50 \mu \mathrm{M}$ ML265 (Cayman Chemical; CAS 1221186-53-3) was added to the cultures. Dorsomorphin $(2 \mu \mathrm{M})$ (Cayman Chemical; CAS 866405-64-3) was used to inhibit BMP4. Recombinant BMP4 $(10 \mathrm{ng} / \mathrm{ml})$ was purchased from PeproTech (120-05ET). To block the glycolytic pathway, 5 mM 2DG (Sigma-Aldrich; S8375) was added to the culture medium. To block PD-L1 (CD274), purified anti-human CD274 antibody $(10 \mu \mathrm{g} / \mathrm{ml}$; BioLegend; 329710, 29E.2A3) or isotype control antibody $(10 \mu \mathrm{g} / \mathrm{ml}$; BioLegend; 401212, MG2b-57) were included in the culture

Probably the most important observation made in this study relates to the metabolic regulation of PD-L1 expression, identifying nutrient oversupply as an upstream checkpoint in macrophage biology (summarized in Figure 10). Excess uptake of glucose and loading of the glycolytic intermediate pyruvate into the mitochondria enhances the proinflammatory functions of CAD macrophages, but also drives up their expression of PD-L1. Thus, metabolic conditions, specifically overnutrition, ultimately regulate $\mathrm{T}$ cell immunity. The correction of glucose uptake and normalization of the levels and subcellular distribution of cellular metabolites may provide a novel therapeutic access to the regulation of adaptive immune system functions.

\section{Methods}

Patients and controls. The study population included 113 patients with CAD who were selected as previously described (24). Patients who had at least 1 documented myocardial infarction were enrolled more than 90 days after an ischemic event. Their demographic characteristics are summarized in Table 1. Demographically matched healthy individuals were recruited from the Stanford Blood Center $(n=109)$. These patients had no personal or familial history of autoimmune disease, cancer, chronic viral infection, or any other chronic inflammatory disorder. medium for the indicated durations (Figures 6 and 7). Sodium pyruvate (5 mM) was purchased from Sigma-Aldrich (P8574), and $2 \mu$ M UK5099 (Calbiochem; 5.04817.0001) was added to the culture medium to inhibit mitochondrial pyruvate import.

Macrophage-T cell cocultures. T cell-stimulatory functions of maclated with LPS and IFN- $\gamma$ for 24 hours and loaded with $1.5 \mathrm{ng} / \mathrm{ml}$ antiCD3/OKT antibody (BioLegend) for 1 hour. CD $4 \mathrm{~T}$ cells were purified and mixed with macrophages at a ratio of 1:5 in 96-well U-bottomed plates. In selected experiments, the ratio was changed as indicated in each figure legend.

Quantitative reverse transcription PCR. Total RNA was extracted with Direct-zol RNA MiniPrep (Zymo Research), and cDNA was reverse transcribed using the High-Capacity cDNA Reverse Transcription Kit (Thermo Fisher Scientific). Gene expression was determined using $2 \times$ SYBR Green qPCR Master Mix (Biotool.com) and a RealPlex2 Mastercycler (Eppendorf) as previously reported (24). The primers used are listed in Supplemental Table 1. Gene transcript numbers were adjusted relative to $\beta$-actin transcripts.

Flow cytometry. Surface molecule expression was measured using a BD LSRFortessa, and data were analyzed with FlowJo software (Tree Star). The following antibodies were obtained from BioLegend: allophycocyanin-conjugated (APC-conjugated) anti-CD25 (302610, BC96); rophages were tested in a coculture system. Macrophages were stimu- 


\section{Table 1. Demographics of patients with CAD}

\begin{tabular}{|cc|}
\hline No. of patients & 113 \\
\hline Age, yr (mean \pm SD) & $69.9 \pm 8.8$ \\
\hline Male & $92.0 \%$ \\
\hline Race & \\
\hline White & $85.0 \%$ \\
\hline African American & $5.3 \%$ \\
\hline Asian & $8.8 \%$ \\
\hline BMI (mean \pm SD) & $30.4 \pm 4.9$ \\
\hline Diabetes mellitus & $49.6 \%$ \\
\hline Hypertension & $70.8 \%$ \\
\hline Hyperlipidemia & $75.2 \%$ \\
\hline Family history of CAD & $61.1 \%$ \\
\hline Smoking history & \\
\hline Current & $10.6 \%$ \\
\hline Former & $61.9 \%$ \\
\hline Treatment & \\
\hline ACE inhibitor & $54.0 \%$ \\
\hline Angiotensin II receptor blocker & $17.7 \%$ \\
\hline -Blocker & $57.5 \%$ \\
\hline Ca antagonist & $35.3 \%$ \\
\hline Diuretic & $40.7 \%$ \\
\hline Antiplatelet & $79.6 \%$ \\
\hline Statin & $84.1 \%$ \\
\hline Sulfonylurea & $8.8 \%$ \\
\hline Insulin & $20.3 \%$ \\
\hline
\end{tabular}

Pacific Blue-conjugated anti-CD69 (310920, FN50); APC-conjugated anti-CD45RA (304112, HI100); FITC-conjugated anti-CD45RO (304204, UCHL1); APC-conjugated anti-CD274 (PD-L1, 329708, 29E.2A3); phycoerythrin-conjugated (PE-conjugated) anti-CD273 (PDL2, 329606, 24F.10C12); PE-conjugated anti-CD4 (317410, OKT4); and Pacific Blue-conjugated anti-CD86 (305423, IT2.2). To evaluate cytokine production in T cells, the cells were restimulated with $50 \mathrm{ng} /$ $\mathrm{ml}$ PMA and $1 \mu \mathrm{g} / \mathrm{ml}$ ionomycin (Life Technologies, Thermo Fisher Scientific) for 8 hours, permeabilized using the BD Cytofix/Cytoperm Kit, stained with PE-conjugated anti-IFN- $\gamma$ (BD; 554552 and 13780) and FITC-conjugated anti-IL17A (BioLegend; 512304 and BL168), and analyzed by flow cytometry. GolgiStop (BD; 564219) was added for the last 6 hours of culture. To evaluate intracellular protein in macrophages, the cells stimulated with LPS and IFN- $\gamma$ for 24 hours were fixed, permeabilized, stained with rabbit anti-IRF1 (Santa Cruz Biotechnology Inc.; sc-497) or rabbit anti-p-SMAD1/5 (Cell Signaling Technology), stained with PE-conjugated goat anti-rabbit antibody (Santa Cruz Biotechnology Inc.; sc-3739), and analyzed by flow cytometry.

Cell proliferation assay. Total CD4 T cells were labeled with CellTrace CFSE (Life Technologies, Thermo Fisher Scientific) and cocultured with M1 macrophages for 6 days. Next, the cells were washed, stained with PE-conjugated anti-CD4 and APC-conjugated antiCD45RA, and analyzed by flow cytometry. Representative figures show CD4-gated cell populations. Proliferation indices (division number of responding cells) were calculated with FlowJo software.

siRNA-mediated knockdown. To knock down PD-L1 expression, $1 \mathrm{nM}$ siRNA oligonucleotides were transfected into macrophages using INTERFERin (Polyplus Transfection). Oligo duplex RNA specific for PD-L1 and a negative control were purchased from GE Dharmacon. Forty-eight hours after transfection, macrophages were stimulated and tested.

Measurement of IFN- $\gamma$-secreting VZV-specific T cells. ELISPOT assays were performed as described previously (6). Briefly, serial dilutions of PBMCs were cultured in duplicate on precoated anti-IFN- $\gamma$ ELISPOT plates (Mabtech) and stimulated with $3 \mu \mathrm{g} / \mathrm{ml} \mathrm{VZV} \mathrm{viral} \mathrm{lysate} \mathrm{for}$ 18 hours. Spots were developed with an HRP-conjugated anti-INF- $\gamma$ antibody and tetramethylbenzidine (TMB) substrate. Plates were analyzed using ImmunoSpot software (Cellular Technology Ltd.).

IHC. IHC was performed using previously published protocols (24). Briefly, OCT-embedded sections of carotid endarterectomy specimens were sectioned at $10-\mu \mathrm{m}$ intervals and fixed with $4 \%$ paraformaldehyde solution (Affymetrix). Formalin-fixed, paraffin-embedded tissue samples were deformalinized with xylene and ethanol, and antigen retrieval was performed using Target Retrieval Solution (Dako; S1699). After blocking with 5\% normal goat serum (Invitrogen, Thermo Fisher Scientific), sections were incubated with unconjugated primary antibody overnight. Primary antibodies included mouse antihuman CD68 antibody (1:200; Dako; M0814, KP1); rabbit anti-human CD68 antibody (1:200; Santa Cruz Biotechnology Inc.; sc-9139); rabbit anti-BMP4 antibody (1:200; Abcam; ab124715); and mouse anti-PD-L1 (1:200; BioLegend; 329702, 29E.2A3). Antibody binding was visualized by staining with Alexa Fluor 488-conjugated goat antirabbit IgG (Life Technologies, Thermo Fisher Scientific; A11008) or Alexa Fluor 546 goat anti-mouse IgG (Life Technologies, Thermo Fisher Scientific; A11003) secondary antibodies for 2 hours and mounted with DAPI-containing mounting medium (Molecular Probes). Tissue sections were examined using fluorescence microscopy.

Statistics. Statistical significance was calculated by unpaired or paired, 2-tailed Student's $t$ test, Mann-Whitney $U$ test, 1-way ANOVA, or 2-way ANOVA as appropriate. All analyses were performed using GraphPad Prism Version 7 (GraphPad Software). For all analyses, $P$ values of less than 0.05 were considered significant.

Study approval. The study was approved by the IRB of Stanford University, and written informed consent was obtained from all study participants.

\section{Author contributions}

RW and TS planned and performed experiments and were responsible for data analysis. HN, HZ, and BS contributed technical expertise, and DGH participated in the conceptional development of the project. BBW enrolled patients in the study, and JAT and JCG oversaw patient recruitment. GJB supervised tissue analysis. CMW and JJG conceived the study, designed experiments, and analyzed data, and CMW, JJG, and RW wrote the manuscript.

\section{Acknowledgments}

This work was supported by the NIH (R01 AR042547, R01 HL117913, R01 AI108906, and P01 HL129941, to CMW, and R01 AI108891, R01 AG045779, and I01 BX001669, to JJG). RW received fellowship support from the Cahill Discovery Fund.

Address correspondence to: Cornelia M. Weyand, Department of Medicine, Stanford University School of Medicine, CCSR Building Room 2225, 269 Campus Drive West, Stanford, California 943055166, USA. Phone: 650.723.9027; Email: cweyand@stanford.edu. 
1. Arvin A. Aging, immunity, and the varicella-zoster virus. $N$ Engl J Med. 2005;352(22):2266-2267.

2. Heininger U, Seward JF. Varicella. Lancet. 2006;368(9544):1365-1376.

3. Haberthur $\mathrm{K}$, et al. CD4 T cell immunity is critica for the control of simian varicella virus infection in a nonhuman primate model of VZV infection. PLoS Pathog. 2011;7(11):e1002367.

4. Kawai K, Gebremeskel BG, Acosta CJ. Systematic review of incidence and complications of herpes zoster: towards a global perspective. BMJOpen. 2014;4(6):e004833.

5. Johnson RW. Herpes zoster and postherpetic neuralgia. Expert Rev Vaccines. 2010;9(3 Suppl):21-26

6. Qi Q, et al. Diversification of the antigen-specific $\mathrm{T}$ cell receptor repertoire after varicella zoster vaccination. Sci Transl Med. 2016;8(332):332ra46.

7. Qi Q, et al. Defective T memory cell differentiation after varicella zoster vaccination in older individuals. PLoS Pathog. 2016;12(10):e1005892.

8. Wang C, et al. B-cell repertoire responses to varicella-zoster vaccination in human identical twins. Proc Natl Acad Sci U S A. 2015;112(2):500-505.

9. Jones L, Black AP, Malavige GN, Ogg GS. Phenotypic analysis of human $\mathrm{CD} 4+\mathrm{T}$ cells specific for immediate-early 63 protein of varicella-zoster virus. Eur J Immunol. 2007;37(12):3393-3403.

10. Schub D, et al. Altered phenotype and functionality of varicella zoster virus-specific cellular immunity in individuals with active infection. J Infect Dis. 2015;211(4):600-612.

11. Vukmanovic-Stejic M, et al. The characterization of varicella zoster virus-specific $\mathrm{T}$ cells in skin and blood during aging. J Invest Dermatol. 2015;135(7):1752-1762.

12. Criqui MH, Aboyans V. Epidemiology of peripheral artery disease. Circ Res. 2015;116(9):1509-1526.

13. Koskinen J, et al. Effect of age, gender and cardiovascular risk factors on carotid distensibility during 6-year follow-up. The cardiovascular risk in Young Finns study. Atherosclerosis. 2012;224(2):474-479.

14. Vigen R, Maddox TM, Allen LA. Aging of the United States population: impact on heart failure. Curr Heart Fail Rep. 2012;9(4):369-374.

15. Joesoef RM, Harpaz R, Leung J, Bialek SR. Chronic medical conditions as risk factors for herpes zoster. Mayo Clin Proc. 2012;87(10):961-967.

16. Ke CC, et al. Increased risk of herpes zoster in diabetic patients comorbid with coronary artery disease and microvascular disorders: A population-based study in Taiwan. PLoS One. 2016;11(1):e0146750.

17. Wu PY, Lin CL, Sung FC, Chou TC, Lee YT. Increased risk of cardiovascular events in patients with herpes zoster: a population-based study. JMed Virol. 2014;86(5):772-777.

18. Hansson GK, Hermansson A. The immune system in atherosclerosis. Nat Immunol. 2011;12(3):204-212.

19. Crea F, Liuzzo G. Pathogenesis of acute coronary syndromes. J Am Coll Cardiol. 2013;61(1):1-11.

20. Libby P, Lichtman AH, Hansson GK. Immune effector mechanisms implicated in atherosclerosis: from mice to humans. Immunity. 2013;38(6):1092-1104.

21. Witztum JL, Lichtman AH. The influence of innate and adaptive immune responses on atherosclerosis. Annu Rev Pathol. 2014;9:73-102.

22. Libby P, Tabas I, Fredman G, Fisher EA. Inflammation and its resolution as determinants of acute coronary syndromes. Circ Res. 2014;114(12):1867-1879.

23. Rogacev KS, et al. CD14++CD16+ monocytes independently predict cardiovascular events: a cohort study of 951 patients referred for elective coronary angiography. J Am Coll Cardiol. 2012;60(16):1512-1520.

24. Shirai T, et al. The glycolytic enzyme PKM2 bridges metabolic and inflammatory dysfunction in coronary artery disease. JExp Med. 2016;213(3):337-354.

25. Palsson-McDermott EM, et al. Pyruvate kinase M2 regulates Hif- $1 \alpha$ activity and IL-1 $\beta$ induction and is a critical determinant of the Warburg effect in LPS-activated macrophages. Cell Metab. 2015;21(1):65-80.

26. Chaneton B, Gottlieb E. Rocking cell metabolism: revised functions of the key glycolytic regulator PKM2 in cancer. Trends Biochem Sci. 2012;37(8):309-316.

27. Christofk HR, Vander Heiden MG, Wu N, Asara JM, Cantley LC. Pyruvate kinase M2 is a phosphotyrosine-binding protein. Nature. 2008;452(7184):181-186.

28. Chen L, Flies DB. Molecular mechanisms of T cell co-stimulation and co-inhibition. Nat Rev Immunol. 2013;13(4):227-242.

29. Legein B, Temmerman L, Biessen EA, Lutgens E. Inflammation and immune system interactions in atherosclerosis. Cell Mol Life Sci. 2013;70(20):3847-3869.

30. Woo SR, Corrales L, Gajewski TF. Innate immune recognition of cancer. Annu Rev Immunol. 2015;33:445-474.

31. Brahmer JR, et al. Safety and activity of antiPD-L1 antibody in patients with advanced cancer. N Engl J Med. 2012;366(26):2455-2465.

32. Garon EB, et al. Pembrolizumab for the treatment of non-small-cell lung cancer. $N$ Engl J Med. 2015;372(21):2018-2028.

33. Hamid $O$, et al. Safety and tumor responses with lambrolizumab (anti-PD-1) in melanoma. $N$ Engl JMed. 2013;369(2):134-144.

34. Chen J, Jiang CC, Jin L, Zhang XD. Regulation of PD-L1: a novel role of pro-survival signalling in cancer. Ann Oncol. 2016;27(3):409-416.

35. Chinai JM, Janakiram M, Chen F, Chen W, Kaplan M, Zang X. New immunotherapies targeting the PD-1 pathway. Trends Pharmacol Sci. 2015;36(9):587-595.

36. Zou W, Wolchok JD, Chen L. PD-L1 (B7-H1) and PD-1 pathway blockade for cancer therapy: Mechanisms, response biomarkers, and combinations. Sci Transl Med. 2016;8(328):328rv4.

37. Zhang $\mathrm{H}$, et al. Immunoinhibitory checkpoint deficiency in medium and large vessel vasculitis. Proc Natl Acad Sci U S A. 2017;114(6):E970-E979.

38. Atefi M, et al. Effects of MAPK and PI3K pathways on PD-L1 expression in melanoma. Clin Cancer Res. 2014;20(13):3446-3457.

39. Lee SJ, et al. Interferon regulatory factor- 1 is prerequisite to the constitutive expression and IFN-gamma-induced upregulation of B7-H1 (CD274). FEBS Lett. 2006;580(3):755-762.
40. Noman MZ, et al. PD-L1 is a novel direct target of HIF-1 $\alpha$, and its blockade under hypoxia enhanced MDSC-mediated T cell activation. JExp Med. 2014;211(5):781-790.

41. Yao S, et al. Control of pathogenic effector T-cell activities in situ by PD-L1 expression on respiratory inflammatory dendritic cells during respiratory syncytial virus infection. Mucosal Immunol. 2015;8(4):746-759.

42. Walsh MJ, et al. ML265: A potent PKM2 activator induces tetramerization and reduces tumor formation and size in a mouse xenograft model. In: Probe Reports from the NIH Molecular Libraries Program. Bethesda (MD): National Center for Biotechnology Information (US); 2010.

43. Ford ML, Adams AB, Pearson TC. Targeting co-stimulatory pathways: transplantation and autoimmunity. Nat Rev Nephrol.2014;10(1):14-24.

44. Smyth MJ, Ngiow SF, Ribas A, Teng MW. Combination cancer immunotherapies tailored to the tumour microenvironment. Nat Rev Clin Oncol. 2016;13(3):143-158.

45. Hayashi Y, et al. BMP-SMAD-ID promotes reprogramming to pluripotency by inhibiting $\mathrm{p} 16 /$ INK4A-dependent senescence. Proc Natl Acad Sci U S A. 2016;113(46):13057-13062.

46. Katagiri T, Watabe T. Bone morphogenetic proteins. Cold Spring Harb Perspect Biol. 2016;8(6):a021899.

47. Yadin D, Knaus P, Mueller TD. Structural insights into BMP receptors: Specificity, activation and inhibition. Cytokine Growth Factor Rev. 2016;27:13-34.

48. Alves-Filho JC, Pålsson-McDermott EM. Pyruvate kinase M2: A potential target for regulating inflammation. Front Immunol. 2016;7:145

49. Mazurek S. Pyruvate kinase type M2: a key regulator of the metabolic budget system in tumor cells. Int J Biochem Cell Biol. 2011;43(7):969-980.

50. Mazurek S, Boschek CB, Hugo F, Eigenbrodt E. Pyruvate kinase type $\mathrm{M} 2$ and its role in tumor growth and spreading. Semin Cancer Biol. 2005;15(4):300-308.

51. Goronzy JJ, Weyand CM. Understanding immunosenescence to improve responses to vaccines. Nat Immunol. 2013;14(5):428-436.

52. Pereira BI, Akbar AN. Convergence of innate and adaptive immunity during human aging. Front Immunol. 2016;7:445.

53. Duffield JS, Lupher M, Thannickal VJ, Wynn TA. Host responses in tissue repair and fibrosis. Annu Rev Pathol. 2013;8:241-276.

54. Chen D, Zhao M, Mundy GR. Bone morphogenetic proteins. Growth Factors. 2004;22(4):233-241.

55. Csiszar A, Labinskyy N, Jo H, Ballabh P, Ungvari Z. Differential proinflammatory and prooxidant effects of bone morphogenetic protein- 4 in coronary and pulmonary arterial endothelial cells. Am J Physiol Heart Circ Physiol. 2008;295(2):H569-H577.

56. Guo WT, Dong DL. Bone morphogenetic protein-4: a novel therapeutic target for pathological cardiac hypertrophy/heart failure. Heart Fail Rev. 2014;19(6):781-788.

57. Miriyala S, et al. Bone morphogenic protein- 4 induces hypertension in mice: role of noggin, vascular NADPH oxidases, and impaired vasorelaxation. Circulation. 2006;113(24):2818-2825. 
58. Poggio P, et al. Noggin attenuates the osteogenic activation of human valve interstitial cells in aortic valve sclerosis. Cardiovasc Res. 2013;98(3):402-410.

59. Sun B, et al. Bone morphogenetic protein- 4 mediates cardiac hypertrophy, apoptosis, and fibrosis in experimentally pathological cardiac hypertrophy. Hypertension. 2013;61(2):352-360.

60. Wong WT, et al. Bone morphogenic protein-4 impairs endothelial function through oxidative stress-dependent cyclooxygenase-2 upregulation: implications on hypertension. Circ Res. 2010;107(8):984-991.

61. Csiszar A, Lehoux S, Ungvari Z. Hemodynamic forces, vascular oxidative stress, and regulation of BMP-2/4 expression. Antioxid Redox Signal. 2009;11(7):1683-1697.

62. Hong OK, et al. High glucose and palmitate increases bone morphogenic protein 4 expression in human endothelial cells. Korean J Physiol Pharmacol. 2016;20(2):169-175.

63. Koga M, et al. BMP4 is increased in the aortas of diabetic ApoE knockout mice and enhances uptake of oxidized low density lipoprotein into peritoneal macrophages. J Inflamm (Lond). 2013;10(1):32.

64. Feng J, et al. BMP4 enhances foam cell formation by BMPR-2/Smad1/5/8 signaling. Int J Mol Sci.
2014;15(4):5536-5552.

65. Kallioniemi A. Bone morphogenetic protein 4-a fascinating regulator of cancer cell behavior. Cancer Genet. 2012;205(6):267-277.

66. Navi BB, et al. Association between incident cancer and subsequent stroke. Ann Neurol. 2015;77(2):291-300.

67. Wang EY, Dixson J, Schiller NB, Whooley MA. Causes and predictors of death in patients with coronary heart disease (from the Heart and Soul Study). Am JCardiol. 2017;119(1):27-34.

68. De Vlaeminck Y, González-Rascón A, Goyvaerts C, Breckpot K. Cancer-associated myeloid regulatory cells. Front Immunol. 2016;7:113. 